\title{
VIII. DIE WITWE
}

1. „Essayer de mettre ordre aus affayres« Anna und der Streit um die Thronfolge

In den Monaten vor der Eröffnung der Ständeversammlung zu Blois hatten sich die Machtverhältnisse in Frankreich so verschoben, daß der König, welcher noch kurz zuvor ein vorteilhaftes Heiratsprojekt für Annas Sohn unverwirklicht gelassen hatte, nun gezwungen war, einige der anderen Wünsche seiner cousine zu erfüllen. Dazu gehörte, neben der finanziellen Entschädigung fur die geplatzte Hochzeit des Herzogs und dessen Emennung zum Gouverneur von Lyon, die Bemühung beim Papst um die Übertragung der Legation von Avignon an den Kardinal von Guise. Während die Prinzessin diesbezuglich schon Jahre zuvor furr ihren Sohn eingetreten war, wandte sich Heinrich nun persönlich an Sixtus V. mit der Bitte, den Kardinal auf besagten Posten zu berufen. Damit war ein weiteres der Ziele erreicht, auf die Anna jahrelang zugesteuert hatte. Parallel dazu trat Katharina von Medici an der Kurie wieder einmal für die Ernennung des Marquis von Saint-Sorlin zum Kardinal ein ${ }^{1}$. Der Groll des Königs gegen die italienische Prinzessin muß folglich groß gewesen sein, de Thou ist sogar der Ansicht, sie habe im Dezember 1588 um ihr Leben zu fürchten gehabt. Es ist jedoch unwahrscheinlich, daß sich die Enkelin Ludwigs XII. tatsächlich in ernsthafter Gefahr befunden hat, ein Augenzeuge des Gesprächs zwischen Heinrich und seiner Mutter kurz nach der Ermordung des Herzogs von Guise berichtet vielmehr, der König habe bekräftigt, um Annas Treue seiner Person gegenüber zu wissen, und angeblich nur aus Sicherheitsgrunden Wachen vor der Tür ihres Gemachs postiert ${ }^{2}$.

\footnotetext{
${ }^{1}$ Anna d'Este an Alfonso und Luigi d'Este, o.D. (Ende Nov.-Anfang Dez. 1585), in: ASM, Canc. duc., Cart. prin. est. 1458/22, 1459/23; Alfonso d'Este an Luigi d'Este, 14. Dez. 1585, Ferrara, in: ASM, Casa 84; Heinrich III. an Sixtus V., 15. Nov. 1588, Blois, in: Richard COOPER, The Blois Assassinations: Sources in the Vatican, in: Keith CAMERON (Hg.), From Valois to Bourbon. Dynasty, State and Society in Early Modern France, Exeter 1989, S. 5172, hier S. 63f.; Katharina von Medici an Sixtus V., an den Marquis de Pisany, französischer Gesandter in Rom, und an Alessandro Peretti, Kardinal von Montalto, 15. u. 17. Nov. 1588, Blois, in: MÉDICIS, Lettres, Bd. 9, S. 388-390.

${ }^{2}$ Filippo Cavriana an Antonio Serguidi, 24. Dez. 1588, in: DESJARdINS (Hg.), Négotiations diplomatiques, Bd. 4, S. 843; THOU, Histoire universelle, 1740, Bd. 7, S. 344.
} 


\section{Blois 1588 und die Klage der trauernden Mutter}

Über die Möglichkeit eines Anschlags auf das Leben ihres ältesten Sohnes war die Herzogin schon informiert, bevor sie im Herbst 1588 nach Blois reiste. Zwei ihrer Besucher hatten sie auf die bestehende Gefahr aufmerksam gemacht: Étienne Pasquier und Jacques-Auguste de Thou, dem gegenüber sie sogar eine dustere Vorahnung geăußert haben soll ${ }^{3}$. In der Nacht vor dem Attentat scheint Anna dann, in Trănen aufgelöst, dem Herzog berichtet zu haben, man wolle ihn am nächsten Tag im Kabinett des Königs ermorden, eine Szene, welche die Phantasie französischer Autoren bis ins 20. Jahrhundert hinein angeregt hat ${ }^{4}$. Am frühen Morgen des 23. Dezember präsentierte sich Henris Sekretär Jean Péricard, der aus den uber dem Vorzimmer der Königinmutter liegenden Răumen verdächtigen Lărm vernommen hatte, vor der Prinzessin und flehte sie an, sich Katharina vor die Füße zu werfen, damit sich diese zu Heinrich begebe, pour détourner cet orage. Daraufhin schickte Anna einen ihrer Vertrauten zu Katharina und einen anderen zum päpstlichen Legaten mit der Nachricht, daß sie um das Leben ihrer Söhne fürchte und der Bitte, sich für sie einzusetzen. Doch weder dem Kirchenmann noch der Herzogin selbst sollte es gelingen, beim König oder seiner Mutter vorzusprechen. Als Anna ihr Gemach verlassen wollte, fand sie ein Dutzend Schweizer vor ihrer Tür postiert, die den Befehl hatten, niemanden hinein oder heraus zu lassen'.

Die Prinzessin blieb nur kurze Zeit eingesperrt, spätestens am 27. Dezember konnte sie sich wieder frei im Schloß bewegen und empfing die Besuche Katharinas von Medici, der Damen des Hofes, der ferraresischen Agenten und einiger Kapuzinermönche ${ }^{6}$. Noch während ihrer Haft hatte Anna durch einen Boten der Königinmutter vom Tod ihres ältesten Sohnes erfahren. Als dann

${ }^{3}$ PASQUIER, Lettres historiques, S. 362f.; THOU, Histoire universelle, 1740, Bd. 7, S. 348.

${ }^{4}$ Relatione di quel che successo in Bles, Ende Dez. 1588, in: COOPER, Blois Assassinations, S. 67. Dustere Vorahnung und vergebliche Warnung der Prinzessin werden in "Le Guysien " thematisiert, einer Tragodie von Simon Belyard (1592 gedruckt, aber vorher bereits auf der Bühne): Louis LOBBES, L'exécution des Guises, prétexte à tragédie, in: Yvonne BELLENGER (Hg.), Le mécénat et l'influence des Guise. Actes du Colloque de Joinville 1994, Paris 1997, S. 567-579, ebenso wie in der Erzählung "L'assassinat du duc de Guise« von Pierre Lafue, Paris 1942, S. 158f.

${ }^{5}$ Aussage von Jean Péricard und Victor Le Pelletier vor der Kommission zur Untersuchung der Morde an Henri und Louis de Lorraine, 3. u. 28. März 1589, in: BnF, Fr. 17300, S. 226228, 275f., Zitat S. 227; Giovanni Francesco Morosini, Nuntius in Frankreich, an einen unbekannten Empfänger in Florenz, Jan. 1589, in: DESJARDINS (Hg.), Négotiations diplomatiques, Bd. 4, S. 868. Wahrend des Arrests durfte Anna niemanden sehen, außer zwei bis drei ihrer Diener: Avviso aus Blois, 24. Dez. 1588, in: COOPER, Blois Assassinations, S. 71.

${ }^{6}$ Galeazzo Fiaschi an Alfonso d'Este, 26.-27. Dez. 1588, Saint-Dyé-sur-Loire, in: ASM, Canc. duc., Amb. Francia 98; Bernardino de Mendoza an Philipp II., 27. Dez. 1588, SaintDyé-sur-Loire, in: Croze, Les Guises, Bd. 2, S. 387. Vgl. den Brief vom 20. Jan. 1589, La Chaussée-Saint-Victor, in: AN, 21 Mi 157: Madame de Nemurs no tiene guardas, pero está se como prisionera. 
noch die Nachricht eintraf, auch der Kardinal von Guise sei auf Geheiß Heinrichs umgebracht worden, war die Herzogin sprachlos vor Schmerz. Dem Bruder schrieb sie:

Mes afflictions sont si extrêmement grandes et déplorables que, n'aiant ny la force ny le caur de les pouvoir escrire, $j$ 'ay prié le $s$ 'Rondinelli de les représenter à V.A., auquel je vous supplie trèshumblement de croire comme elle feroit à moy mesme, et à ce coup avoir pitié de vostre paure, desolée seur et servante et $m$ 'ayder de vostre bon conseil et aide ${ }^{?}$.

Nichts sollte in den folgenden Monaten die Gemüter der Zeitgenossen so erregen wie der Gedanke an die Verzweiflung und Trauer der Mutter. Zwei diesbezügliche Texte wurden als Pamphlete gedruckt und verbreitet: Die "Remonstrance faicte par madame de Nemours à Henry de Valloys « und die "Regrets de madame de Nemours sur la mort de Messeigneurs de Guyse ses enfans" ${ }^{8}$. Während in den "Regrets" die angebliche Sorge der Herzogin um das Königreich behandelt wird, beinhaltet die »Remonstrance« eine Anklage gegen die Grausamkeit und Tyrannei des französischen Herrschers. Es ist unwahrscheinlich, daß diese Schrift einen tatsächlich an den Konig adressierten Brief darstellt, und auch die Behauptung, Heinrich habe Anna aus Wut über dieses Schreiben einsperren lassen, kann in das Reich der Legenden verwiesen werden'. Vielmehr handelt es sich hierbei um politische Pamphlete, verfaßt von Sympathisanten der Ligue, um die Bevölkerung der Hauptstadt zum Widerstand gegen einen ungeliebten König aufzurufen; sie als wirkliche Briefe oder gar genuine literarische Arbeiten zu verstehen, die mit denen einer Margarete von Navarra in einem Atemzug genannt werden könnten, ist irreführend $^{10}$. Über dreißig verschiedene Drucke und Drucksammlungen, welche die aktuellen Ereignisse auf meist polemische Weise thematisieren, sind aus der Zeit nach der Ermordung der Guise-Brüder und dem Zusammenbruch der königlichen Autorităt uberliefert, darunter auch diese beiden die Mutter der

7 Anna d'Este an Alfonso d'Este, 25. Dez. 1588, Blois, in: ASM, Canc. duc., Cart. prin. est. 1458/22. Ercole Rondinelli war ehemals Vikar von Luigi d'Este in Frankreich.

${ }^{8}$ Vgl. Denis Pallier, Recherches sur l'imprimerie à Paris pendant la Ligue (1585-1594), Genf 1975, Nr. 308, 309, 311. Zu Druck und Verbreitung von politischen Schriften zur Zeit der Ligue: Jean QUÉNIART, Les Français et l'écrit, Paris 1998, S. 63-67.

${ }^{9}$ Mit dieser Geschichte kommentierte ein Kapuzinermönch aus Lyon die italienische Ubersetzung der "Remonstrance«, welche von ihm nach Rom geschickt wurde: COOPER, The Aftermath, S. 409. Hier auch die Behauptung, bei der »Remonstrance« würde es sich um einen wirklichen Brief handeln.

${ }^{10}$ So in: Susan BROOMHALL, Women and the Book Trade in Sixteenth-Century France, Aldershot, Burlington 2002, S. 122. Zum Verständnis der Pamphlete als von den Fürstinnen tatsăchlich verfaßte Werke (»works «): Ibid. S. 97-99 (hinsichtich eines angeblichen Briefes der Herzogin von Mayenne an ihren Gemahl), und Colette H. WINN, Ecriture, veuvage et deuil. Témoignages féminins du XVI siècle, in: Nicole PELLEGRN, DiEs. (Hg.), Veufs, veuves et veuvage dans la France d'Ancien Régime, Paris 2003, hier S. 286 (hinsichtlich der »Regrets et lamentations« der Herzogin von Guise). 
Verstorbenen betreffenden Schriften ${ }^{11}$. So wird der Text der "Remonstrance«, nicht anders als die "Regrets«, noch zur Zeit ihrer Haft und ohne Wissen der Prinzessin in Paris entstanden sein, wobei die Verfasser aus propagandistischen Gründen aber durchaus Bilder benutzten, derer sich Anna während des Prozesses gegen die Mörder ihres ersten Gemahls bedient hatte. Vor allem die Strategie der Demonstration von Schwäche und Stärke zugleich ist zu erkennen.

Auf dem Titelblatt der »Regrets« ist das Portrait einer Dame in Trauerkleidung abgebildet (Abb. 6). Das Motiv der trauernden Mutter dient hier dem gleichen Zweck wie das der trauernden Witwe fünundzwanzig Jahre zuvor: Es stellt die für die Anklage notwendige Legitimation bereit und schafft einen Eindruck von Verletzlichkeit und Schwäche. Annas Selbstinszenierung während ihres großen Prozesses damals scheint sich den Parisern so ins Gedächtnis gebrannt zu haben, daß sie es als ganz natürlich ansahen, die Prinzessin in Witwentracht auf dem Titel ihrer Pamphlete abzubilden, während die Herzogin von Guise auf einer vergleichbaren Schrift im Ballkleid dargestellt ist, obwohl auch sie bereits ihren zweiten Gemahl verloren hatte und außerdem schwanger war (Abb. 7). Der Text der »Remonstance« beginnt mit den Fragen und der Ratlosigkeit der trauernden Mutter und somit aus einer defensiven Haltung heraus:

Je ne sçay (Roy cruel) depuis quel temps tu es devenu si aveuglé, que tu ne veois pas à te conduire. Je ne sçay (tiran inhumain) quelle serpentine et bourrelle rage c'est emparee de ton caur, d'avoir usé de tant de cruautez envers mes enfans, qu'il n'est possible de plus. Quelle offence ont ils comis envers toy, de les avoir faict massacrer de coups à tes yeux? ${ }^{12}$

Doch dann werden die Ermordeten als diejenigen dargestellt, qui ont mis la courronne sur ton chef, t'ont mis le sceptre en main et ont preservé ton heritage, die aux depens de leur sang et de leurs biens gegen die Hugenotten gekämpft und, assistez de la Majesté divine, die reîtres vertrieben hätten. Die Legitimităt der Herrschaft Heinrichs wird hier in Frage gestellt, welche, nicht anders als die seines Bruders Karls, allein auf dem persönlichen Engagement der Guise beruhe, nicht nur auf dem des Sohnes, sondern auch auf dem des Vaters: mon mary par trahison, à un service si mal recompencé perdit le premier la vie. Wie ein Bogen spannt sich die Argumentation vom Mord an François zum Tod von Henri und Louis, die alle im Dienst für die Valois ihre Leben gelassen hätten.

\footnotetext{
${ }^{11}$ Philip BENEDICT, Of Marmites and Martyrs. Images and Polemics in the Wars of Religion, in: French Renaissance in Prints, S. 108-137, hier S. 127.

${ }^{12}$ Les Cruautez sanguinaires, exercées envers feu Monseigneur le Cardinal de Guise, Pair de France et Archevesque de Reims. Avec la remonstrance faicte au Roy par Madame la Duchesse de Nemours, sur le massacre de ses enfans, Paris 1589, S. 11. Die folgenden Zitate: Ibid. S. 11 f. Vgl. MUNNS, RICHARDS, Exploiting, S. 212.
} 
In diesem Zusammenhang erklären sich auch die sechs mit »Regrets funebres de tres-haute et tres-illustre princess Anne d'Este (à present Duchesse de Nemours) aux ombres de tres-haut et tres-excellent Prince François de Lorraine, Duc de Guyse, son mary « überschriebenen Sonette in den »Premières cuvres poétiques « des Flaminio de Birague, welche in die Ausgaben von 1583 und 1585 Eingang gefunden haben. Zwei Jahrzehnte nach dem Attentat wird hier die Trauer der Witwe wieder in das Gedächtnis der Zeitgenossen gerufen und an die Verdienste ihres Gemahls um die Christenheit erinnert. Mit der rhetorischen Frage an den Schutzengel des Hauses Lothringen: Ne prevoyoistu pas que nostre sainte foy / Auroit encor besoin de ce grand demi-Roy [...]? wird eine Parallele zwischen den Taten des großen Herzogs François und der Beteiligung der Guise-Brüder an der Ligue gezogen, die somit als Kampf furr das Königreich interpretiert werden konnte ${ }^{13}$. Aus jenen Jahren stammt auch ein Vierzeiler mit dazugehörigem Porträt, der die Prinzessin, dargestellt in der obligatorischen Witwentracht, als Beschützerin der durch den großen Sturm hin- und hergeworfenen französischen Lilie beschreibt: Cette plante voiant une grande tempeste / Qui faisoit Chanceller deça dela le lis / Fleurit pour l'appuyer, de trois genereux fils / Sans le secours desquels il n'eust peu faire teste (Abb. 8) ${ }^{14}$. Die Anklage Annas in der »Remonstance« von 1589 jedenfalls gipfelt in der an den König gerichteten Bitte um ihren eigenen Tod: je te prie, fay moy mourir avec mes enfans, car c'est ce que le plus je desire, ce faisant, de Roy cruel et inhumain que tu es, je diray que tu seras doux et courtois ${ }^{15}$.

Welche Popularität das Motiv der trauernden Mutter genoß, ist neben den zahlreichen Nachdrucken dieser Propagandaschriften an der Verbreitung abzulesen, welche die Szene auch in bildlicher Form fand. Auf einem mit "Comme les deux Princes, estans morts, sont mis sur une table« betitelten Druck sind die nackten Leichen der Guise-Brüder zu sehen, aus deren Wunden noch das Blut tropft:

Madame de Nemours, voyant ses deva fils

Si malheureusement et perfidement occis,

Se print à escrier, disant: O Malheureux

Barbare, inhumain, est-ce la récompense

De ceux qui te maintenoient possible en France?

Fais m'en donc autant, je gise avec eux! ${ }^{16}$

${ }^{13}$ Sonett III der »Epitaphes«, in: BIRAGUE, Les premières œuvres, Bd. 3, S. 161.

${ }^{14}$ BnF, Fr. 13764, fol. 8r. Inhaltlich bezieht sich der Vierzeiler auf die Jahre 1589-1595, das Bildnis einer Dame, bei dem es sich um ein wahres Porträt Annas handeln könnte, kann auf den Anfang des 17. Jh. datiert werden.

${ }^{15}$ Cruautez sanguinaires, S. 12.

${ }^{16}$ Comme les deux princes estans morts sont mis sur une table, avec la remonstrance de madame de Nemours, à Henry de Valloys, et l'emprisonnement de messieurs les princes catholicques, Paris 1589, in: Pierre de L'EsTolLE, Mémoires-Journaux (1574-1611), Bd. 4: 
Sowohl das Motiv der trauernden Witwe und Mutter, die den Tyrannen um den Tod bittet, als auch die Behauptung, Heinrich habe seine Regierung den Guise zu verdanken, ist von Pierre Matthieu in seiner die Ereignisse verarbeitenden "Guisiade" wieder aufgenommen worden, wo die Klage der Herzogin lautet: Tu l'as donc massacré? massacre aussi sa mere, / Qui veut accompagner, et le fils et le pere, / Les tuteurs de la France: et sans eux tu n'aurois / Le sceptre, l'ornement, et l'honneur de nos Roys. Wahrscheinlich hat der Autor die »Remonstrance« und die bildliche Darstellung des Geschehens für sein Stück benutzt, ebenso wie die zahlreichen Gerüchte, die über das Schicksal der Körper der Getöteten kursierten, denn er lăßt Anna ausrufen: Las! me permettront-ils ces bourreaux qu'en grand dueil, / Pour office dernier je te mette au cercueil? ${ }^{17}$

Tatsächlich hatte die Herzogin einen ihrer Besucher, den Kapuzinermönch Bernardo d'Osimo, zur Königinmutter geschickt mit der Bitte, sich für die Herausgabe der Leichname einzusetzen. Katharina beruhigte den Mönch und trug ihm auf, die Freundin zu trösten und ihr zu versichern, daß die Körper ihrer Sơhne beigesetzt worden seien. Als Bernardo wieder im Gemach der gefangenen Prinzessin eintraf, rief diese, man habe ihr erzählt, die Leichen seien verbrannt worden. Er hätte sie zwar gerne vom Gegenteil ubberzeugt, resümiert der Mönch seine Reaktion, ma in effetto era pur troppo vero. Tatsächlich ist aus einem unmittelbar nach den Ereignissen verfaßten Bericht zu erfahren, daß die Königinmutter auf die Frage nach dem Leichnam des Herzogs von Guise die Antwort erhalten habe, daß Heinrich den Körper zwar noch verwahre, ma che ne haveva di besogno. Daraufhin soll der Herrscher einen Boten zu seiner Gefangenen gesandt haben, welcher ihr par de très grands serments versicherte, daß die Verstorbenen fort honorablement in geweihter Erde beigesetzt worden seien ${ }^{18}$. Die Vorstellung von der trauernden Mutter, die den Tyrannen um die Herausgabe ihres toten Kindes bittet, hat die Zeitgenossen unglaublich fasziniert. Die Szene wurde viele Male verarbeitet, wobei Anna auf verschiedenste Weise dargestellt wurde: Von der sich verzweifelt vor dem Herrscher auf die Knie werfenden Trauernden bis hin zu der stolzen Dame, die, sans s'amuser à répandre des larmes, [...] alla droit au

Les belles figures et drolleries de la ligue (1589-1600), hg. v. G. BRUNET u.a., Paris 1876, ND 1982, S. 35f. Eine Abb. in: LelouP, Anne d'Este, S. 26. Vgl. PalliER, Recherches, Nr. 281. Zu den Nachdrucken: BroOMHALL, Book Trade, S. 122.

${ }^{17}$ Pierre MATTHEU, La Guisiade, hg. v. Louis LOBBES, Genf 1990, S. 164f. Die Widmung der Tragödie an den Bruder der Ermordeten ist auf den 1. Mai 1589 datiert.

${ }^{18}$ Discorso d'un capuccino che diede gran danno all' interessi del signor cardinale Morosini legato in Francia, in: C. VALOIS ( $\mathrm{Hg}$.), Histoire de la Ligue. Guvre inédite d'un contemporain, Bd. 1: 1574-1589, Paris 1914, S. 295-304, hier S. 299-301, Zitat S. 301; Um 1620 niedergeschriebener Bericht eines Zeitgenossen, in: Ibid. S. 279; Relatione di quel che successo in Bles, Ende Dez. 1588, in: COOPER, Blois Assassinations, S. 68. 
Roi. Doch nur einen Ring, den der Verstorbene am Finger getragen hatte, gestand Heinrich der Prinzessin $\mathrm{zu}^{19}$.

\section{Der weibliche Gefangene des Königs}

Nicht weniger bewegt hat eine weitere Episode jener Wochen die Gemüter der Zeitgenossen: Die Inhaftierung der Herzogin von Nemours im Schloß von Amboise. Der Grund für die Entscheidung des Königs, entgegen eines ursprunglichen Versprechens außer seinen männlichen Gefangenen auch Anna nach Amboise zu schaffen, ist sicher davon beeinflußt worden, daß diese sich gleich nach der Aufhebung ihres Zimmerarrests gemeinsam mit einer anderen Dame an der Verbreitung umstürzlerischer Gerulchte beteiligt hatte ${ }^{20}$. Wie bemerkenswert die Tatsache war, daß neben einem Kardinal, einem Erzbischof, mehreren adligen Herren und einigen weiteren Männern auch Anna unter den Ende Januar 1589 nach Amboise verlegten Inhaftierten war, ist aus einem an den spanischen König gerichteten Brief zu ersehen, in dem es heißt: el rey en persona havía ydo a Amboissa, castillo fuerte, donde llevó consigo a Madama de Guisa [!] y otro prisioneros. Der Verfasser irrt sich zwar hinsichtlich der Person oder ihres Titels, läßt aber die Namen der betroffenen Herren unerwähnt ${ }^{21}$. Der Nuntius, welcher der Gefangenen zwei Wochen zuvor in Blois noch einen Besuch abgestattet hatte, wandte sich, als er von der drohenden Verlegung erfuhr, sofort an den König und versuchte, ihn mit dem Hinweis auf Unschuld und Treue der Herzogin von seinem Vorhaben abzubringen. Heinrich antwortete, Anna müsse zwar gemeinsam mit den anderen an einen sichereren Ort gebracht werden, per tenere i figlioli in frena, e non per mala volontà che li portasse, daß sie aber in camere assai ampli wohnen würde und empfangen durfe, wen sie wolle ${ }^{22}$. Wăhrend in Ferrara zu erfahren war,

${ }^{19}$ Die erste Darstellung: Histoire au vray du meurtre et assassinat perfidement et proditoirement commis au cabinet du Roy, o.D. (Ende 16. Jh.), in: BnF, Fr. 5795, fol. 50v. Die zweite und das Zitat: THOU, Histoire universelle, 1740, Bd. 7, S. 348. Zu dem Ring: Aussage von Jacques des Vignes vor der Kommission zur Untersuchung der Morde an Henri und Louis de Lorraine, 18. Febr. 1590, in: BnF, Fr. 17300, S. 278.

${ }^{20}$ Bernardino de Mendoza an Philipp II., 31. Dez. 1588, Saint-Dyé-sur-Loire, in: CROZE, Les Guises, Bd. 2, S. 389; Febo Bonnà an Alfonso d'Este, 3. Febr. 1589, Paris, in: ASM, Canc. duc., Amb. Francia 98. Bei der anderen Dame handelt es sich um die Maréchale de Joyeuse, Marie de Batarnay. Ein weiterer Grund für die Inhaftierung Annas in Amboise liegt wohl in der Tatsache, daß dem Herzog von Nemours die Flucht aus Blois gelungen war.

${ }^{21}$ Juan de Canoza an Philipp II., 18. Febr. 1589, Venedig, in: AN, 21 Mi 248. In den zeitgenössischen Aufzählungen der Verhafteten wird Anna meist an erster Stelle genannt: VIENNOT, Femmes d'État, S. 81. Die Gefangenen wurden Ende Januar nach Amboise verlegt, nicht, wie vielerorts behauptet, Ende Dezember.

${ }^{22}$ Galeazzo Fiaschi an Alfonso d'Este, 16. Jan. u. 6. Febr. 1589, Vendôme, Blois, in: ASM, Canc. duc., Amb. Francia 98. 
die Prinzessin trüge diesen weiteren Schicksalsschlag con meravigliosa fortezza d'animo, wurde nach Florenz berichtet: Le lagrime che si sono sparse da quei poveri prencipi, quando furono condotti là non si possono raccontare, und es war Heinrich höchstpersönlich, der seine Gefangenen die Loire hinab nach Amboise geleitete ${ }^{23}$.

Bei der Abreise aus Blois soll sich folgende Begebenheit zugetragen haben: Die Herzogin von Nemours, bevor sie in das wartende Boot stieg, wandte sich zum Schloß um, über dessen Tor eine Reiterstatue des Erbauers, ihres Großvaters Ludwig XII. zu sehen war, und rief: Monsieur, jamais vous n'avez fait construire ceste maison vostre pour servir de forest ny de massacre à voz petits-enfans ${ }^{24}$. Kein Bericht der Folgezeit, in dem dieser Satz fehlte; Matthieu ließ ihn in seiner Tragödie aufsagen, und die Herzogin von Guise benutzte die Idee in einem Brief an den Papst: Das Schloß sei von König Ludwig errichtet worden, so die Witwe, afin qu'il servist d'un palais vraiement Royal, et non pas d'une spelonque d'assassins pour y massacrer proditoirement ceux qui descendroient de luy en ligne directe ${ }^{25}$. Außer zu Zwecken der Polemik nutzte die königliche Abkunft Anna jedoch nichts, wie die anderen wurde auch sie eingesperrt, doch genoß sie Hafterleichterung und konnte Besuche empfangen. Etwa eine Woche blieb die Herzogin in Amboise, dann wurde sie, ad instanza di molti, freigelassen ${ }^{26}$. Noch in Blois hatte der König ihr befohlen, den Sohn schriftlich zur Niederlegung der Waffen aufzufordern, nun wollte er die Prinzessin dazu benutzen, mit dem Herzog von Mayenne einen Friedensvertrag auszuhandeln, woran man erkennen kann, wie ambivalent Heinrichs Politik in den letzten Monaten seiner Regierungszeit war. Brantôme berichtet, die Zeitgenossen hätten sich über diese Entscheidung entsprechend gewundert, und Anna selbst hätte den Herrscher gar verspottet. Doch nicht nur Heinrich, auch ein italienischer Beobachter war der Ansicht, die Herzogin zähle zu den weni-

${ }^{23}$ Febo Bonnà an Alfonso d'Este, 3. Febr. 1589, Paris, in: ASM, Canc. duc., Amb. Francia 98; Filippo Cavriana an Antonio Serguidi, 9. Febr. 1589, in: DESJARDINS (Hg.), Négotiations diplomatiques, Bd. 4, S. 860.

${ }^{24}$ Le martyre des deux frères, contenant au vray toutes les particularitez plus notables des massacres, et assassinats, commis és personnes de tres-hauts, tres-puissants, et treschrestiens Princes, Messeigneurs le Reverendissime Cardinal de Guyse Archevesque de Reins. Et de Monseigneur le Duc de Guyse Pairs de France (1589), in: L. CimBER, Félix DANJOU (Hg.), Archives curieuses de l'histoire de France depuis Louis XI jusqu'à Louis XVIII, 1. Ser., Bd. 12, Paris 1836, S. 57-107, hier S. 105.

${ }^{25}$ MATTHIEU, La Guisiade, S. 165; Catherine de Clèves an Sixtus V., 17. Febr. 1589, Paris, in: COOPER, The Aftermath, S. 424.

${ }^{26}$ Filippo Cavriana an Antonio Serguidi, 9. Febr. 1589, in: DESJARDINS (Hg.), Négotiations diplomatiques, Bd. 4, S. 860; Galeazzo Fiaschi an Alfonso d'Este, 8. u. 10. Febr. 1589, Vendôme, in: ASM, Canc. duc., Amb. Francia 98. 
gen Personen, die die Schwierigkeiten in Frankreich senza sangue lösen könn$\operatorname{ten}^{27}$.

Wenige Wochen später war die Hoffnung auf Frieden vernichtet, und Anfang März 1589 hieß es, Heinrich glaube nicht mehr, daß die Herzogin noch etwas erreichen könne, por las muchas dificultades [que] nasceran de una y otra parte $^{28}$. Die Erleichterung über die Freilassung der Prinzessin war jedenfalls groß. In Annecy wurden Freudenfeuer gezündet, und Alfonso d'Este versicherte dem Herzog von Savoyen, il mondo sei auch vorher schon si chiaro dell' innocenza sua gewesen, daß es keiner weiterer Zeugen bedurft habe ganz so, als wäre seine Schwester eines wirklichen Verbrechens angeklagt gewesen. Anna selbst schrieb dem Bruder: an mes estrêmes annuys yl m'est arrivé cest heur que le Roy, après mon emprisonemant, m'a remise an liberté et permis d'aller où ye voudroys, und bot im Namen de se quy me reste d'anfans ihre Dienste an ${ }^{29}$. Die Stadt Paris hatte am Schicksal der Prinzessin regen Anteil genommen und sie zu einer Heldin, zur Mutter eines Märtyrers stilisiert. Während L'Estoile auf der Straße den Ausruf: $O$ saint et glorieux martyr de Dieu, béni est le ventre qui t'a porté et les mamelles qui t'ont allaité, gehört zu haben meinte, ließ Belyard in seiner Tragödie den Tyrannen zu der Trauernden sagen, am liebsten hätte er sie gezwungen, die Leichen ihrer Söhne zu verspeisen: Affin que ce tien ventre, ou ils furent conceus, / Fust tombeau des Enfans qui de luy sont issus ${ }^{30}$.

Über die Taten der Herzogin von Nemours in den Monaten nach ihrer Freilassung schweigen die Quellen, ein Vorgehen gegen den König scheint sie nicht ernsthaft in Erwägung gezogen zu haben. Matthieu läßt sie in seiner Tragödie den Herrscher verfluchen: Ton cueur, ton lasche cueur aux plus

${ }^{27}$ Relazione del sig. Manelli, secretario dell' ill ${ }^{\text {mo }}$ cardinal Gondi, partito di Francia alli xi et arrivato in Praga a xxii di febraro 1589, in: BnF, Fr. 5045, fol. 180f., hier fol. 180v; BRANTÓME, Recueil des Dames, S. 709; Filippo Cavriana an Antonio Serguidi, 9. Febr. 1589, in: DESJARDINS (Hg.), Négotiations diplomatiques, Bd. 4, S. 861f. Vgl. Palma CAYET, Chronologie novenaire, Bd. 2, S. 6: Heinrich III. habe Anna freigelassen avec la charge d'enhorter les princes ses enfans, ses parens, et tous autres à son obeyssance.

28 Juan de Canoza an Philipp II., 4. Mărz 1589, Venedig, in: AN, 21 Mi 248. BRANTÔME, Recueil des Dames, S. 708f. beschreibt mit viel Phantasie das Treffen zwischen Anna und dem Herzog von Mayenne und behauptet, die Prinzessin habe ihre Mission zwar erfullt, unter der Hand dem Sohn aber ihre wahre, keineswegs friedfertige Meinung erläutert.

${ }^{29}$ Alfonso d'Este an Carlo Emanuele di Savoia, 24. Febr. 1589, Ferrara, in: AST (Cast.), Int., Let. div., Prin. for. 30; Anna d'Este an Alfonso d'Este, 12. Febr. 1589, Paris, in: ASM, Canc. duc., Cart. prin. est. 1458/22. Vgl. Lucrezia d'Este an Francesco Maria della Rovere, 1. März 1589, Ferrara, in: ASF, DU, cl. I, 112. Zum Freudenfeuer: Register der Beschlüsse des Stadtrats von Annecy, 16. Febr. 1589, in: AMA, BB 11, vol. 23, fol. 85r.

${ }^{30}$ L'EsTolle, Journal Henri III, S. 606, mit falscher Angabe des Datums; Simon Belyard, Le Guysien ou perfidie tyrannique commise par Henry de Valois ès personnes des illustriss. reverendiss. et tresgenereux Princes Loys de Loraine cardinal, et Archevesque de Rheims, et Henry de Loraine Duc de Guyse, grand Maistre de France, Troyes 1592, zit. n. LOBBES, L'exécution des Guises, S. 576. 
paisibles nuits / Sera outré d'horreurs, de trances, et d'ennuis: / Car mon Dieu justicier ne lairra impunie / Ta fiere cruauté, ta blesme tyrannie, und Pasquier beschuldigt sie, gemeinsam mit anderen Damen der Familie für das Attentat auf Heinrich verantwortlich zu sein. Brantôme wirft der Prinzessin zwar keine direkte Beteiligung daran vor, doch berichtet er, sie habe nach den Ereignissen in Blois den König nur noch als ce tyran bezeichnet und mit ihren angeblichen Rachegelüsten zu dem Mord beigetragen: Une Dame de grand coeur qui couve une vindicte est fort à craindre, wie er sich ausdrückt. L'Estoile geht einen Schritt weiter und behauptet, Anna sei nach Erhalt der Nachricht vom Tod des Herrschers durch die Straßen von Paris gefahren, um dem Volk die Bonnes nouvelles mitzuteilen, sie habe Freudenfeuer zünden und Feste feiern lassen und von den Treppen eines Altars herab eine Rede gehalten, montrant en cet acte une grande immodestie et impuissance de femme ${ }^{31}$. Ihr diesbezüglicher, an den Bruder adressierter Brief hört sich jedoch recht nüchtern an:

Il me semble que je ferois faulte à mon debvoir si je manquois à vous donner advis que du jour d'hier, premier de ce mois, le Roy fut blessé sur les dix heures du matin par ung religieux de l'ordre des Jacobins incogneu, aagé de vingt quatre ans, d'ung coup de couteau au dessoubs du petit ventre. Or, à jourd'hry sur les trois heures du matin, est mort. Ce porteur en rendra plus au long conte à Votre A., qui me gardera vous estre plus longue ${ }^{32}$.

Möglich ist auf jeden Fall, daß Anna die Witwe ihres ermordeten Sohnes bei ihrem juristischen Vorgehen gegen den König unterstützte. Bereits Ende Januar war von der Herzogin von Guise beim Parlament ein Gesuch eingereicht worden, welches um die Untersuchung des Falles bat. In welchem Maße sich Catherine dabei an der von ihrer Schwiegermutter fünfundzwanzig Jahre zuvor benutzten Strategie orientierte, ist an ihrem an den Papst gerichteten Brief abzulesen. Dort heißt es: ie me prosterne par ceste humble lettre aux piedz de vostre Saincteté, pour crier lamentablement, d'une voix que ie desirerois estre oüye des quatre coings du monde, IUSTICE, IUSTICE, IUSTICE; [...] IUSTICE à une vefve desolee et à neuf petis enfans orphelins, womit die Witwe

${ }^{31}$ MATthIEU, La Guisiade, S. 165; Estienne PASQuIER, L'Anti-Martyr (1590), in: DerS., Écrits politiques, hg. v. Dorothy THICKETT, Genf 1966, S. 179-246, hier S. 220f.; BRANTÓME, Recueil des Dames, S. 705f.; Pierre de L'EsTonL, Journal pour le règne de Henri IV, Bd. 1: 1589-1600, hg. v. Louis-Raymond LefÉVRE, Paris 1948, S. 19. Vgl. Barbara B. DIEFENDORF, An Age of Gold? Parisian Women, the Holy League, and the Roots of Catholic Renewal, in: Michael WOLFE (Hg.), Changing Identities in Early Modern France, Durham, London 1997, S. 169-190, hier S. 172f.

${ }^{32}$ Anna d'Este an Alfonso d'Este, 2. Aug. 1589, Paris, in: ASM, Canc. duc., Cart. prin. est. $1458 / 22$. 
Annas Kniefall sowie ihren Ruf nach Gerechtigkeit aufnahm und die damals mit Erfolg gefluhrte Kampagne wiederzubeleben suchte ${ }^{33}$.

\section{Verwaltung und Repräsentation im Namen der Ligue}

In der Zeit zwischen der Ermordung Heinrichs III. und der Anerkennung seines Nachfolgers durch die Herzöge von Mayenne und von Nemours, die zu den letzten Rebellen gegen den neuen König gehörten, spielte Anna d'Este eine zentrale Rolle fur das politische Geschehen in Frankreich. Anfang Januar 1589 , als sie im Schloß von Amboise gefangen saß, war Katharina von Medici gestorben, so daß die Prinzessin nun, dank der Macht ihrer Söhne, die vielleicht einflußreichste Dame des Konigreichs war. Darliber hinaus hielt sie sich, von wenigen Ausnahmen abgesehen, die ganze Zeit über in der Hauptstadt auf, wo in jenen Jahren wichtige Entscheidungen getroffen wurden und wo sie sehr beliebt war. Von der Ligue zur »Königinmutter" ernannt, erfullte Anna tatsächlich Aufgaben, die einer regierenden Königin zugekommen wären. Sie nahm an den Sitzungen des als "Staatsrat" bezeichneten Gremiums teil, die manchmal sogar in ihrem Haus abgehalten wurden, und sie empfing offizielle Besucher. Quartier bezog sie standesgemäß im Hôtel de la Reine, der ehemaligen Wohnung der Katharina von Medici in der Pariser Rue des DeuxÉcus, und zwar in den Gemächern, die einst die Prinzessin von Lothringen, Katharinas Enkelkind, bewohnte. Im Sommer 1589, kurz nachdem die Herzogin ihr neues Domizil bezogen hatte, schmückten noch diejenigen Gemälde die Wănde, welche auf Geheiß der Königinmutter angebracht worden waren; lediglich ein Porträt ihrer Schwester, der Herzogin von Urbino, könnte von Anna stammen. Im Laufe der Zeit ließ die Prinzessin den logis de la royne renovieren, säubern und einrichten, etwa mit ihrem pettit cabinet, das im November 1591 aus dem Hôtel de Nemours in die Rue des Deux-Écus gebracht wurde. Während der gesamten Zeit der Herrschaft der Ligue scheint Anna zwischen ihrem eigenen Haus und dem der Katharina von Medici gependelt zu sein, da sie nicht gewillt war, einen derart symbolträchtigen Ort wie das Hôtel der Königinmutter aufzugeben ${ }^{34}$.

In unruhigen Zeiten boten sich Frauen besonders gute Möglichkeiten zur Partizipation am politischen Tagesgeschehen, wie das Beispiel niederländischer Witwen zur Zeit des Unabhängigkeitskrieges zeigt: Wăhrend die Măn-

${ }^{33}$ Catherine de Clèves an Sixtus V., 17. Febr. 1589, Paris, in: COOPER, The Aftermath, S. 426. Vgl. die Requeste presenté à Messieurs de la Court de Parlement de Paris, (31. Jan.) 1589, in: AN, 21 Mi 157.

${ }^{34}$ Rechnungsbücher 1591, fol. 39r, 1593-II (Sept.). Vgl. Edmond BONNAFFÉ, Inventaire des meubles de Catherine de Médicis. Meubles, tableaux, objets d'art, manuscrits, Paris 1874, ND 1973, S. 30, 38; MICHAHELLES, Inventory, S. 32. 
ner mit militärischen Aktionen beschäftigt waren, förderten die Frauen das Vorankommen ihrer Familien. Auch die Bemühungen der Herzogin von Nemours galten in erster Linie dem Wohl ihres Nachwuchses. Soweit ihre eigenen politischen Ansichten in den Quellen überhaupt zu erkennen sind, scheint es, als habe Anna die Ligue zwar anfangs unterstützt, sei dann aber zum Frieden und zur Anerkennung Heinrichs IV. bereit gewesen. Bereits im Sommer 1589 berichtete der spanische Gesandte, die Herzogin arbeite an einer Einigung mit dem König, wobei sie darauf bedacht sei, durch vorteilhafte Heiratsprojekte den größtmöglichen Nutzen für ihre Kinder und Enkelkinder herauszuschlagen, und tatsächlich versuchte die Prinzessin spätestens seit Heinrichs erneuter Konversion zum Katholizismus, ihre Söhne in diesem Sinne zu beeinflussen ${ }^{35}$.

Die Bedeutung, welche Annas Anwesenheit in der Hauptstadt während der Belagerung durch Heinrich IV. für die Pariser hatte, ist schon allein aus der Tatsache zu ersehen, daß ihr zwei die Ereignisse schildernde Texte gewidmet sind: die Beschreibung eines anonymen Verfassers, bei dem es sich wahrscheinlich um Gabriel Chappuys handelt, und die französische Übersetzung einer vergleichbaren Schrift des Spaniers Pedro Cornejo ${ }^{36}$. Von den sich zwischen Mai und August 1590 in Paris aufhaltenden Damen, so Filippo Pigafetta in einem weiteren, Gregor XIV. gewidmeten Bericht, Madame di Nemurs era la primiera ${ }^{37}$. Zwei Begebenheiten verraten den Einfluß, welchen die Belagerten in Paris der Prinzessin zugestanden. Als Anfang August der Staatsrat über

${ }^{35} \mathrm{Zu}$ dem durch den spanischen Gesandten verbreiteten Gerücht: Raymond RITTER, La Scur d'Henri IV: Catherine de Bourbon, 1559-1604, Bd. 1, Paris 1985, S. 428. Zu den niederländischen Witwen: Sherrin Marshall WYNTJES, Surviviors and Status: Widowhood and Family in the Early Modem Netherlands, in: Journal of Family History 7 (1982) S. 396-405, hier S. 404.

${ }^{36}$ Adolphe DUfOUR, Histoire du siège de Paris sous Henri IV en 1590 d'après un manuscrit nouvellement découvert, in: Mémoires de la Société de l'histoire de Paris et de l'lle-deFrance 7 (1880) S. 175-270. Chappuys war Literat und Übersetzer im Umkreis der Großen des Hofes und lange Zeit von den Guise protegiert. Der Text von Cornejo auf Französisch: Pierre CORNEJO, Discours bref et veritable des choses plus notables arrivées au Siege mèmorable de la renommée Ville de Paris et défense d'icelle, par Monseigneur le Duc de Nemours contre le Roi de Navarre, Paris 1590, in: Mémoires de la Ligue, contenant les évenemens les plus remarquables depuis 1576, jusqu'à la Paix accordée entre le Roi de France et le Roi d'Espagne, en 1598, Nouvelle édition, Bd. 4, Amsterdam 1758, S. 276-303. Eine moderne Übersetzung: Pedro CORNEJO, Relation sommaire et véridique des choses dignes de remarque arrivées pendant le siège mémorable de la fameuse ville de Paris, et sa défense par le duc de Nemours, contre Henri de Bourbon, prince de Béarn, prenant le titre de roi de France et de Navarre. Traduit de l'espagnol, Paris 1834, die Widmung S. 3. Die spanische Originalversion erschien am 17. Sept. 1590, die französische Übersetzung kurz darauf bei Didier Millot in Paris. Vgl. PaLlier, Recherches, Nr. 708. Anna wird auch erwähnt in der »Histoire« des Nicolas Brûlart, vgl. LE PERSON (Hg.), Journal d'un ligueur.

${ }^{37}$ Filippo PIGAFETTA, Relatione dell' assedio di Parigi, Rom 1591, S. 29. Vgl. AUBIGNÉ, Histoire universelle, Bd. 8, S. 176: Il y avoit une autre sorte de garnison qui fomentoit merveilleusement les resolutions de ce peuple, c'estoyent les Dames. 
die Aufnahme von Friedensverhandlungen mit dem Navarresen debattierte, wurden Abgeordnete zu ihr geschickt, den Stand der Verhandlungen mitzuteilen und ihre Ratschläge entgegenzunehmen; die folgenden Sitzungen wurden dann direkt im Hôtel de Nemours abgehalten. Außerdem soll Heinrich der Herzogin Briefe geschrieben und Boten gesandt haben mit dem Ziel, sie zur Beeinflussung ihres Sohnes zu seinen Gunsten zu überreden. Keiner dieser angeblichen Briefe hat sich erhalten, ihre Erwähnung in den Berichten der Beobachter zeigt aber, daß die Ligue die Prinzessin als eine der Personen betrachtete, die Einfluß auf die Ereignisse zu nehmen in der Lage waren ${ }^{38}$. Daruber hinaus wird das Ansehen, welches die Herzogin zur Zeit der Belagerung in Paris genoß, auch an folgendem, von Pigafetta geschilderten Ereignis deutlich: An einem Tag der Friedensverhandlungen war es der Bevölkerung während einer Feuerpause möglich, die Stadt zu verlassen und außerhalb des Belagerungsringes Freunde und Verwandte zu besuchen, et Madama di Nemurs stava sù la cortina del baloardo con le Principesse che furono visitate da molti Signori. Hier erscheint Anna tatsächlich als eine Königin, die in Begleitung der Prinzessinnen ihres Gefolges die Huldigungen der Besucher entgegennimmt ${ }^{39}$.

Die positive Darstellung der Herzogin von Nemours in diesen Schriften rührte sicher auch von der Tatsache her, daß sie die erste war, die nach Aufhebung der Belagerung für die Versorgung der Stadtbevölkerung mit Eßbarem sorgte, und das, obwohl sie zuvor gezwungen gewesen war, zur Ernährung ihrer eigenen Leute einige ihrer Wertgegenstände zu verpfänden. Bereits am 1. September erreichte aus Dourdan ein erster Konvoi Paris, acompagné de bonnes troupes de cheval et grande quantité de vivres. Die Lebensmittel kamen aus einem Ort, an dem Anna das Nutzrecht besaß, und der Zug wurde angefuhrt von einem in ihren Diensten stehenden Ferraresen. $O$ che festa fecea il popolo in veder quasi tutt' un giorno entrare nell' affamata Città Carra, Cavalli, et altri giumenti [...] cariche d'ogni maniera d'alimenti, beschreibt Pigafetta die Begeisterung der ausgehungerten Menschen. Kein Wunder, daß die Prinzessin zur Heldin stilisiert wurde ${ }^{40}$. Sobald die erste Not jedoch behoben war, begann Anna wieder, an ihre eigenen Interessen zu denken. Bei ihrem Sohn, dem Herzog von Mayenne, beschwerte sie sich, sein Halbbruder habe die ihm aus den Kassen der Stadt Paris zustehende Rente nicht erhalten, und der Herzog mußte seine Mutter um ihre Zustimmung zur Verschiebung der Zahlungen auf einen späteren Zeitpunkt bitten, à fin de réserver au pauvre peuple ceste partie qui pourra soullager infinies personnes en la grande

${ }^{38}$ DUFOUR, Histoire du siège, S. 231f., 245, 250, 254; CORNEJO, Discours bref et veritable, S. 295; DERS., Relation sommaire, S. 31; PIGAFETTA, Relatione, S. 62.

${ }^{39}$ Ibid. S. 61.

${ }^{40}$ Rechnungsbuch 1593-II (Nov.); DufouR, Histoire du siège, S. 259; PIGAFETTA, Relatione, S. 85. 
nécessité en laquelle ilz sont réduitz. Das Volk, fugte er hinzu, vous en aura à jamais obligation et tiendra ce bien et secours de vous seule ${ }^{41}$.

Nach der Belagerung der Hauptstadt stieg Annas Bedeutung fur das Tagesgeschehen stetig. In den Jahren 1591 und 1592 nahm sie wiederholt an den Sitzungen des Staatsrates teil, von denen manche wieder im Hôtel de Nemours abgehalten wurden, sie wurde von den Abgeordneten um ihre Meinung gebeten und redete bei den Wahlen der Magistrate mit. Die Boten, welche Anna sowohl aus der Auvergne, aus Lyon, Rouen und Bourges empfing, als auch die, welche sie mindestens zweimal im Monat zu ihren Söhnen schickte, zeigen, wie tief die Prinzessin in das Geschehen der Zeit involviert war ${ }^{42}$. Der Herzog von Mayenne ernannte sie im Herbst 1591, mit einem Pergament, das wie eine königliche Urkunde aufgemacht war, zur Gouverneurin von Montargis, Nemours und einigen anderen Orten, mit der Begrilndung, sie sei diejenige, welche par droict et raison diesen Provinzen die größte amitié und affection entgegenbrächte, die außerdem immer sagement et vertueusement handelte, pour son grand zèlle de religion et pour l'affection qu'elle porte au royaume et principallement audit pays. Die Prinzessin nahm ihre Aufgabe ernst, bemühte sich um die Sicherheit ihrer Schutzbefohlenen und schrieb zahlreiche Briefe, in denen sie um geeignete Maßnahmen bat, afin d'éviter la ruyne qu'ils peuvent ancourir ${ }^{43}$. Ihr Einfluß auf den Sohn war groß, was an einem seiner Schreiben abgelesen werden kann, verfaßt, nachdem ihm von der Mutter eine bestimmte Person fur einen Posten empfohlen worden war: je donnerai tousjours tant de lieu à ce qui me sera recommandé de vostre part que ceux qui s'addresseront à moy, avec la faveur de voz lettres, ne pourront retourner qu'avec tout le contentement qui dépendra de ma disposition ${ }^{44}$.

Daneben erfüllte Anna die repräsentativen Aufgaben einer Königin. Im Frühjahr 1592, als der Prinz von Parma, Ranuccio Farnese, in Begleitung des Herzogs von Guise nach Paris kam, empfing sie den Sohn dessen, der Heinrich zur Aufgabe der Belagerung gezwungen hatte, wie einen hohen Staatsgast. Während die Pariser Ratsherren den beiden jungen Männem noch entge-

${ }^{41}$ Charles de Lorraine an Anna d'Este, 6. Dez. 1590, Du camp de Guise, in: Charles de Lorraine, duc de MAYENNE, Correspondance, sur le manuscrit de la bibliotheque de Reims, hg. v. Edouard HENRY, Charles LORIQUET, in: Travaux de l'Académie impériale de Reims 29 (1858-1859) S. 109-444, hier S. 215.

${ }^{42}$ GUÉRIN u.a. (Hg.), Registres des délibérations, Bd. 10, S. 153f. (26. Aug. 1591), $287 f$. (15. Aug. 1592); L'EsTolle, Journal Henri IV, Bd. 1, S. 139 (20. Nov. 1591); Rechnungsbilcher 1591, 1593-II.

${ }^{43}$ Emennung von Anna d'Este zur Gouverneurin von Montargis, Nemours, Châteaurenard und Lorris durch Charles de Lorraine, 30. Sept. 1591, Originalpergament, in: AST (Cast.), Int., PGN, cat. 9, m. 1, n. 17; Anna d'Este an Louis de Gonzaga, Herzog von Nevers, 3. Okt. 1592, 19. Sept. u. 29. Okt. o.J. (1592-1593), Paris, in: BnF, Fr. 4709, fol. 161r, 165r, Fr. 4718, fol. 79r.

44 Charles de Lorraine an Anna d'Este und Henri de Savoie, 17. Febr. 1591, Soissons, in: MAYENNE, Correspondance, Bd. 33, S. 73. 
gengehen wollten, waren diese schon längst am Hôtel de Nemours angelangt, wo sie die Herzogin begrußten und wo es von Menschen wimmelte: $y$ avoit du monde innumerable, tant en la court que en la chambre de lad. dame, pour le monde qui y alloict de toutes partz de la Ville - die Pariser hatten sich ihren eigenen kleinen Königshof geschaffen ${ }^{45}$. Kein Wunder also, daß die in der Stadt stationierten neapolitanischen und spanischen Truppen bei der Nachricht von der Flucht des Herzogs von Guise aus seinem Gefängnis im Schloß von Tours nicht seiner Mutter gratulierten, sondern der Großmutter. Und noch nach der Konversion des Königs gingen die Hoffräulein seiner Schwester, als sie in die Stadt kamen, zunächst die Herzogin von Nemours begrußen ${ }^{46}$.

Das politische Geschehen in Frankreich brachte Anna aber nicht nur neue Aufgaben und gesteigertes Ansehen, sie profitierte auch materiell von der Macht ihrer Söhne. So gelang es ihr, nachdem sie den die Renten seines Halbbruders betreffenden Brief des Herzogs von Mayenne erhalten hatte, den Stadtrat zur Auszahlung von fünftausend Écus an Charles-Emmanuel zu bewegen. Gesichert war diese Summe durch die schriftliche Verpflichtung der Prinzessin, das Geld bei Bedarf zurlickzuerstatten. Doch selbst Jahre später war dies noch nicht geschehen, so daß die ihr aus den Kassen der Stadt zustehenden Pensionen vollkommen gestrichen wurden ${ }^{47}$. Ein anderer derartiger Fall wird durch eine vom Herzog von Mayenne ausgestellte Urkunde offenbar, mit der er der Mutter die Wandvertäfelungen des Kapuzinerkonvents in Paris schenkte und ihr deren Verwendung nach eigenem Gutdünen erlaubte ${ }^{48}$.

\section{Der mühsame Weg zum Frieden}

Angesichts dieser Beispiele von Selbstbereicherung ist eine Aussage über Annas politische Anschauungen zu diesem Zeitpunkt schwierig. Aufgrund der Tatsache, daß sowohl ihr Sohn als auch ihr Enkel als mögliche Kandidaten fur den französischen Thron galten, wurde sie in der "Satyre Ménippée« als Royne mere, ou grande mere (in dubio) du Roy futur dargestellt, die sich die Schleppe von Madamoiselle de la Ruë tragen lä $\mathrm{Bt}^{49}$. Für die Gemäßigten war klar, daß die Herzogin von Nemours die Pläne der Ligue guthieß und deren

${ }^{45}$ GUÉRnN u.a. (Hg.), Registres des délibérations, Bd. 10, S. 254 (18. Mai 1592).

${ }^{46}$ L'ESTOLLE, Journal Henri IV, Bd. 1, S. 122 (18. Aug. 1591), 311 (23. Aug. 1593).

47 Verbot an die Schatzmeister von Paris, Anna d'Este und Henri de Savoie ihre Renten auszuzahlen, 28. Aug. 1595, in: GuÉRIN u.a. (Hg.), Registres des délibérations, Bd. 11, S. 159. Vgl. den Hinweis auf Annas Zahlungsverpflichtung, 13. Dez. 1590, in: Ibid. Bd. 10, S. 80, Anm. 2.

${ }^{48}$ Schenkung von Charles de Lorraine an Anna d'Este, 25. Febr. 1592, in: AST (Cast.), Int., PGN, cat. 9, m. 1, n. 18.

${ }^{49}$ E. TRICOTEL (Hg.), Satyre Ménippée de la vertu du Catholicon d'Espagne et de la tenue des Estatz de Paris, Bd. 1, Paris 1877, ND 1971, S. 13. 
radikale Prediger unterstützte, und tatsächlich hatten die Priester ihrer Pfarrei Saint-André-des-Arts und der nicht selten der Volksverhetzung beschuldigte Frère Daniel in den Jahren 1589 bis 1591 wiederholt finanzielle Hilfe von ihr erhalten, letzterer ist in den Monaten Januar bis April 1592 gar als ständiges Mitglied ihres Gefolges in den Ausgabenbüchern verzeichnet ${ }^{50}$.

Erschwert wird eine Aussage über Annas Ansichten dadurch, daß eine der wichtigsten Quellen für jene Jahre die Tagebücher des Pierre de L'Estoile sind, der sich über den Standpunkt der Prinzessin oft selbst nicht im Klaren gewesen zu sein scheint. Zwar stellt er sie meist als zum Frieden und zur Anerkennung des Navarresen bereit dar; diejenigen seiner Anekdoten aber, in denen sie als radikale Verfechterin der Ligue und ihrer Methoden erscheint, sind oftmals so $\mathrm{kraB}$, daß sie das Bild der Herzogin auf nicht unerhebliche Weise geprägt haben. So berichtet L'Estoile, Anna habe einer jungen Frau, die während der Belagerung von Paris über die Hungersnot klagte, geantwortet:

Et quand vous seriez là réduite, que pour votre religion il vous faudrait tuer vos enfants [um sie zu essen], pensez-vous que ce soit si grand cas que cela? De quoi sont faits vos enfants, non plus que cerox de tous les autres: de boue et de crachat! Ma foi, voilà une belle matière pour tant en plaindre la façon! 51

Derselbe Autor erzählt aber auch, die Prinzessin habe sich wiederholt und mit Erfolg bei ihrem Sohn für von der Ligue verfolgte und vertriebene Personen eingesetzt. Im November 1591, nachdem die Seize drei ehemalige Parlamentsmitglieder ermordet hatten, soll Anna dem Herzog einen Boten geschickt haben mit der Bitte, [de] la délivrer elle et sa fille, et tous les gens de bien, de la tyrannie et servitude où ils étaient réduits sous la domination de ces hommes de néant, und, wie es weiter heißt, qu'il se souvînt qu'elle était sa mère, et que c'était celle qui l'avait porté qui l'en priait ${ }^{52}$. Auch hinsichtlich ihrer Bereitschaft zu einer Einigung mit dem König ist L'Estoiles Darstellung ambivalent. In einem Gespräch am Tag vor Heinrichs Rekonversion soll die Herzogin geäußert haben: J'ai apporté ce que j'ai pu pour la paix, mais je n'en ai su venir à bout. J'en suis si contristée que je n'en puis plus, et crois que cela me fera mourir, doch legt er ihr auch das Bekenntnis in den Mund, ihre Bemühungen seien allein dem Zweck gewidmet gewesen, de frapper un

${ }^{50}$ L'EsTOILE, Journal Henri IV, Bd. 1, S. 259 (30. Mai 1593); Rechnungsbuch 1591, fol. 6v (März).

${ }^{{ }^{1}}$ L'ESTOILE, Journal Henri IV, Bd. 1, S. 68f. (19. Aug. 1590). Vgl. auch den Hinweis auf das Mädchen, welches um die Entlassung ihres alten Vaters aus dem Gefängis bat, worauf Anna assez rudement geantwortet habe, daß das nicht möglich sei, pour ce que son père était hérétique et de la religion: L'EsTOILE, Journal Henri III, S. 617 (20. Febr. 1589).

${ }^{52}$ L'Estolle, Journal Henri IV, Bd. 1, S. 99 (1. Apr. 1591), 142 (25. Nov. 1591), 260 (31. Mai 1593). 
grand coup au mariage de mon fils de Nemours avec Madame, der Schwester des Navarresen ${ }^{53}$.

Welche Motive Anna auch gehabt haben mag, ihr Wille zum Frieden ist durch eine große Zahl von Quellen belegt. So berichtete Francesco Panigarola, der sich während der Belagerung in Paris aufgehalten hatte, bereits im Herbst 1590: [Madame de] Nemours est navarroise et n'a autre desir que la paix et que le Navarrois soit Roy, avecq esperance qu'il donne sa sour au duc de Nemours $^{54}$. Einem der Sekretäre des Königs soll sie, nachdem dieser darauf angespielt hatte, daß sie ihre Söhne zu einer Einigung überreden könnte, versichert haben, que mon esprit $y$ travaille, und daß $\mathrm{ihr}$ nichts so sehr am Herzen läge wie der Friede, für den sie alles tun werde, was in ihrer Macht stünde ${ }^{55}$. $\mathrm{Ab}$ dem Sommer 1593 hat die Prinzessin sich dann tatsächlich mit aller Kraft erst für einen Frieden, dann für Heinrichs Anerkennung als König eingesetzt. Inwieweit ihr Bemühen mit dem anderer Damen, die ebenfalls zu einer Abkehr von der Ligue bereit waren, abgestimmt war, ist unklar, möglicherweise bestanden aber Verbindungen zwischen den Fürstinnen in Paris und denen im Süden des Landes ${ }^{56}$. Im August forderte Anna den Herzog von Nemours zur Niederlegung der Waffen auf, erhielt jedoch die Antwort, er stürbe lieber als sich auf einen schändlichen Frieden einzulassen ${ }^{57}$. Nachdem die Pariser Stăndeversammlung beschlossen hatte, einen französischen Fürsten als Gemahl für die Infantin Isabel auszuwählen, um diese dann zu ihrer Königin zu machen, sah man die Prinzessin und ihre Tochter de grands et haults discours mit ihrem Sohn und Bruder Charles führen, avec signes qui démonstroient que l'affaire estoit de grande importance. Die Damen, so der Bericht, widersprachen allem, was sie vom Herzog zu hören bekamen, und behaupteten, sie würden Frankreich eher verlassen, que de permettre ou consentir d'estre Espagnolles $^{58}$.

Das Ereignis, in dessen Folge Anna sich endgültig der Ligue ab- und der Vorantreibung eines Friedens zuwandte, war die Gefangennahme des Herzogs von Nemours in Lyon, wo sich die Stadtbevölkerung im September 1593 der Person des Fürsten bemächtigt und ihn in die Festung Pierre-Scize gespert

${ }^{53}$ L'ESTOILE, Journal Henri IV, Bd. 1, S. 295f. (24. Juli 1593).

${ }^{54}$ Recit de Panigarolle au duc de Savoye de l'estat des affaires de France an l'année 1590, Kopie des 17. Jh., in: BnF, Fr. 4766, fol. 87v. Das italienische Original wurde im Herbst 1590, kurz nach der Aufhebung der Belagerung, verfaBt.

${ }^{55}$ L'ESTonL, Journal Henri IV, Bd. 1, S. 194 (3. Nov. 1592). Zur Bekräftigung seiner Erzăhlung fügt L'Estoile hinzu, er habe seine Informationen de la propre bouche dudit secrétaire.

${ }^{56}$ Vgl. AlLAG, Chrétienne d'Aguerre, S. 132f.

${ }^{57}$ Charles-Emmanuel de Savoie an Anna d'Este, 22. Aug. 1593, Lyon, in: BnF, Fr. 3985, fol. $94 \mathrm{r}$.

${ }_{58}$ Recit des choses qui depuis peu se sont faites à Paris, o.D. (Herbst 1593), in: BnF, Fr. 5045, fol. 411 v (28. Okt. 1593). 
hatte. In einem Brief an die Mutter des Gefangenen versuchten die Lyoner, ihre Tat zu rechtfertigen: Charles-Emmanuel habe sich zum seigneur absolu aufschwingen wollen, weshalb der Stadtrat nun an Annas bonté et naturelle affection que vous avez à ce royaulme, comme iniece ou petite fille de ce bon roy pere du peuple Loys douziesmer appellierte und um Verständnis warb natürlich ohne Erfolg. Die Prinzessin war außer sich: Y'an suis si estrêmemant affligée que ye ne say comme il me sera possible de porter cet annuy, schrieb sie dem Bruder und bat ihn wieder einmal um seine Unterstützung ${ }^{59}$. Die Reaktion der Herzogin auf diese Nachricht läßt erkennen, wie schwer die Ereignisse von Blois auf ihrer Seele lasteten und wie groß die Angst um die Söhne war. Laut L'Estoile soll sie die Schuld an dem Vorfall dem Erzbischof von Lyon gegeben haben, disant mille pouilles de lui et le menaçant, womit eine Verbindung zu dem Mord an Henri de Lorraine hergestellt wäre. Pierre d'Épinac wurde in dessen Folge nämlich vorgeworfen, den Herzog von Guise, der sich damals angeblich aus Blois entfernen wollte, zum Bleiben überredet zu haben, et ce fut pourquoy madame sa mere, wie ihm in der "Satyre Ménippée" in den Mund gelegt wird, $m$ 'a reproché maintefois que i'estoy cause de sa mort. Daneben laßt ein Satz in Annas Brief an den Bruder erkennen, daß sie bei dieser Gefangensetzung auch das Schicksal ihrer beiden ermordeten Sơhne vor Augen hatte: si son frère eust esté avec luy ils seroyent desyà mors tous deus. Die Vorstellung, ihre Kinder dürften sich nie gemeinsam am selben Ort aufhalten, da sich anderenfalls die Ereignisse von Blois wiederholen könnten, scheint in jenen Tagen eine Gewißheit für die Prinzessin geworden zu sein ${ }^{60}$.

In den Monaten nach der Gefangennahme des Herzogs von Nemours unternahm Anna alles nur Mögliche zu seiner Befreiung, drängte ihren Sohn Charles, zugunsten seines Halbbruders einzuschreiten und war bereit, selbst nach Lyon zu reisen und sich persönlich für ihn einzusetzen ${ }^{61}$. Doch obwohl der Marquis von Saint-Sorlin die Gegend um die Stadt herum von seinen Soldaten in Schutt und Asche legen ließ, war eine Freilassung seines Bruders nicht in Sicht. Im Januar schrieb die Herzogin: yusques ici ye n'y voys lumière ny apparanse aucune, und als ganz Paris dartber sprach, daß Heinrichs Trup-

59 Der Lyoner Stadtrat an Anna d'Este, 28. Sept. 1593, Lyon, in: Antoine PÉRICAUD, Notes et documents pour servir à l'histoire de Lyon pendant la Ligue, 1589-1594, Lyon 1844, S. 154-156, Zitate S. 154; Anna d'Este an Alfonso d'Este, 10. Nov. 1593, Paris, in: ASM, Canc. duc., Cart. prin. est. 1458/22. Zu den Ursachen des Konflikts: Robert R. HARDING, Anatomy of a Power Elite. The Provincial Governors of Early Modern France, New Haven, London 1978, S. 105.

${ }^{60}$ L'Estolle, Journal Henri IV, Bd. 1, S. 319 (1. Okt. 1593); Tricotel (Hg.), Satyre Ménippée, S. 28; Anna d'Este an Alfonso d'Este, 10. Nov. 1593, Paris, in: ASM, Canc. duc., Cart. prin. est. 1458/22.

${ }^{61}$ L'Estonte, Journal Henri IV, Bd. 1, S. 321 (25. Okt 1593), 329 (2. Dez. 1593); Anna d'Este an Cario Emanuele de Savoia, 6. Okt. (1593), 9. Jan. (1594), in: AST (Cast.), Int., Let. div., Prin. div. 78. 
pen Orléans eingenommen hătten, winkte sie ab mit dem Hinweis, qu'elle n'a que faire d'Orléans, qu'elle ne songe qu'à Lyon ${ }^{62}$. Ab diesem Zeitpunkt ist in den Quellen kein Hinweis mehr auf eine Unterstutzung der Ligue durch Anna oder auch nur auf gegenseitige Sympathien zu finden, die Prinzessin scheint der Wirren und Unsicherheiten der Zeit endgültig müde gewesen zu sein. Die Flucht sollte Charles-Emmanuel aber erst Ende Juli 1594 gelingen.

\section{Zwischen Kindern und König: Die Versöhnung mit Heinrich IV.}

Obwohl die Herzogin von Nemours an nichts anderes dachte als an ihren Sohn, hatte sie von den Spionen, die sie pour ses intérêts particuliers im Umfeld des Königs unterhielt, von geheimen Verhandlungen zur Übergabe der Hauptstadt an Heinrich erfahren. Anna, qui d'ailleurs ne souhaitoit que la paix, forderte daraufhin den Herzog von Mayenne auf, rechtzeitig mit dem König Frieden zu schließen, aber Charles wollte nicht auf die Mutter hören: il méprisa les avis de la Duchesse de Nemours, comme d'une femme timide, qui ne respiroit que la paix ${ }^{63}$. Die Prinzessin, in der ambivalenten Position zwischen dem eigenen Wunsch nach Frieden und der Loyalität ihren Kindern gegenüber, sollte recht behalten. Kaum einen Monat nach seiner Krönung, die Ende Februar 1594 in Chartres stattgefunden hatte, zog Heinrich in Paris ein. Gleich nach seiner Ankunft sandte er einen Boten zu der wohl wichtigsten Dame der Stadt, sie zu grußen und ihr auszurichten, daß er sie en sa protection et sauvegarde nehmen wolle. Zwei Tage später begab er sich persönlich zum Hôtel de Nemours, um die Herzogin zu besuchen, laquelle se sentit fort obligée à sa Majesté de l'honneur qu'il luy faisoit, et semble que ladite dame soit beaucoup disposée que Messieurs ses deux dernièrs enfans recognoissent sa Majesté $^{64}$. Anna war nun nicht mehr Königinmutter, jetzt war sie es, die sich vom Besuch des Königs geehrt fuhlte und die, als sie nach einer kurzen Reise im April in die Stadt zurlickkehrte, Heinrichs Schwester begrußen ging. L'Estoile berichtet, auch die Bogenschützen und Reiter der Stadt hätten nicht mehr vor der Prinzessin und ihrer Tochter salutiert, sondern die Damen au nez betrachtet und so getan, als kennten sie sie nicht, ce que je ne doute point qu'il ne leur fut un grand crève-cour, principalement à celle qu'on appelait à Paris la Reine-mère. Am 22. Mai 1594 leistete Anna dem König den Treueid, nicht

\footnotetext{
${ }^{62}$ Anna d'Este an Alfonso d'Este, 9. Jan. 1594, Paris, in: ASM, Canc. duc., Cart. prin. est. 1458/22; L'ESTOILE, Journal Henri IV, Bd. 1, S. 370 (20. Febr. 1594).

${ }^{63}$ ThOU, Histoire universelle, 1740, Bd. 8, S. $383 \mathrm{f}$.

${ }^{64}$ L'ESTOILE, Journal Henri IV, Bd. 1, S. 390 (22. März 1594); Particularités de la reddition de Paris et des événements qui l'ont suivie, 25. Mărz (1594), in: BnF, Fr. 5045, fol. 424-426, hier fol. 425r. Eine italienische Übersetzung: DesJaRdins (Hg.), Négotiations diplomatiques, Bd. 5, S. 697.
} 
ohne daß dieser ihr zuvor die Pension bestätigt hätte, die ihr einst von seinem Vorgänger zugesprochen worden war $^{65}$.

In der Folge setzte die Herzogin alles daran, einen Frieden zu erlangen und ihre Kinder zur Anerkennung des Königs zu bewegen. Mit Heinrich hatte sie die Bedingungen für den Übergang von Montargis unter seine Herrschaft ausgehandelt, und im Juni beschloB die dortige Stadtversammlung, ihm den Treueschwur zu leisten ${ }^{66}$. Zur gleichen Zeit berichtete der spanische Beobachter uber Annas Wunsch nach Eintracht und daß sich ihr Sohn über ihre Friedensinitiativen beschwert habe, welche zur Schwächung des Widerstands der Städte gegen den Navarresen beitrugen, que s'il estoit permis de chasser sa mère de sa maison qu'il le feroit ${ }^{67}$. Die Verhandlungen mit dem Herzog von Mayenne und dem Marquis von Saint-Sorlin gestalteten sich entsprechend schwierig, während Charles-Emmanuel noch immer in Lyon gefangen war. Doch auch nach dessen Flucht war keiner der Brüder zur Annäherung an den König bereit, und Anna klagte über l'estrême regret que y'ai que mes anfans ne sont poynt ancores an la bonne grace du Roy. Im Fruhjahr 1595 faßt sie den Plan, gemeinsam mit Heinrich nach Lyon zu reisen, pour essayer de mettre ordre aus affayres. Die so dringend herbeigewünschte Versöhnung ihrer Kinder mit dem Herrscher sei, so die Prinzessin in einem Brief an Alfonso, das Beste für alle, pour le repos de se royaume, le leur, et le bien universe $t^{68}$.

Während Anna in Montargis den königlichen Befehl zur Abreise erwartete, ereilte sie die Nachricht vom Tod des Herzogs von Nemours. Mehr noch als um den Verlust des Sohnes, welcher ihr espérance an mes vieus yours gewesen war, scheint sich die Prinzessin wegen seines jüngeren Bruders Henri und der nun sehr gefährdeten Einigung mit dem König gegrämt zu haben, schrieb sie doch der Schwägerin von ihrem Wunsch, derjenige ihrer Söhne, der ihr noch geblieben sei, möge ihr gehorchen ${ }^{69}$. Charles-Emmanuel war in Annecy

${ }^{65}$ L'Estolle, Journal Henri IV, Bd. 1, S. 413 (23. Apr. 1594), 419 (22. Mai 1594), 427 (15. Sept. 1594); Bestätigung der Pension von 3000 Ecus für Anna d'Este durch Heinrich IV., 3. Mai 1594, in: AST (Cast.), Int., PGN, cat. 9, m. 1, n. 13; Die Urkunde Heinrichs III., 9. Nov. 1574, in: BnF, Fr. 3312, fol. 53r.

${ }^{66}$ Eintrag Nr. 2, sér. BB, vom 15. Juni 1594 im Inventar der Dokumente von Léon Ferré, Notar in Montargis, in: AMM, II, 7, fol. 1r. Vgl. den im Rahmen dieser Einigung erlangten "Arrêt confirmant aux habitants de Montargis la remise des restes du taillon à eux accordéeu, 28. Mai 1594, in: N. VALOIS (Hg.), Inventaire des arrêts du Conseil d'État (règne de Henri IV), Bd. 1, Paris 1886, S. 54f.

${ }^{67}$ Lorenzo Suárez de Figueroa, Herzog von Feria, an Philipp II., 20. Juni 1594, La Fère, Kopie einer zeitgenössischen Übersetzung, in: MC, sér. J, I, fol. 47r.

${ }^{68}$ Anna d'Este an Alfonso d'Este, 14. Febr., 1. Mai, 4. Aug. 1595, Paris, Montargis, in: ASM, Canc. duc., Cart. prin. est. 1458/22. Vgl. Francesco Bonciani an Ferdinando de' Medici, 3. Apr. 1595, in: DESJaRDINs (Hg.), Négotiations diplomatiques, Bd. 5, S. 316.

${ }^{69}$ Anna d'Este an Margherita Gonzaga, Herzogin von Ferrara, o.D. (Ende Aug. 1595), in: ASM, Canc. duc., Cart. prin. est. 1459/23. Vgl. Claude-A. DuCIS, Charles-Emmanuel de Savoie, Duc de Genevois et de Nemours, T. 1, in: Revue savoisienne 23 (1882) S. 53-55, hier S. 54. 
verstorben, im Beisein des Marquis von Saint-Sorlin, der nun den Titel des Herzogs von Nemours trug und der, anstatt Heinrich den Treueid zu leisten, die Flucht ergriffen hatte. Als nämlich Anna, die sich sofort auf den Weg nach Savoyen gemacht hatte, um ihren jüngsten Sohn zu treffen, in Lyon anlangte, mußte sie erfahren, daß dieser Annecy bereits in Richtung Turin verlassen hatte und von dort aus nach Ferrara weiterreisen wollte. Sofort schrieb die Prinzessin ihrem Bruder einen langen Brief, in dem sie bat, Henri zum Gehorsam gegen die Mutter aufzufordern. Da es niemanden auf der Welt gäbe, der ihn so sehr liebe wie sie oder mehr Anteil an seinem Schicksal nehme, müsse sie auch diejenige sein, die den meisten Einfluß und die größte Autorität bei ihm habe. Alfonso solle daher versuchen, de le réduire à me donner la consolation à laquelle Dieu, la nature, et le commandement exprès de feu Monsieur son père l'obligent ${ }^{70}$.

In jenen Wochen war es der Konnetabel, der vor Heinrich die Interessen der Herzogin und ihres jüngsten Sohnes vertrat. Unzählige Male wandte sich die Prinzessin an ihn, schwor, daß Henri zurückkehren, ihr gehorchen und sich in den Dienst des Herrschers begeben würde und bat, dies alles Heinrich wissen zu lassen et nous y favoriser de vos bons offices accoustumes ${ }^{71}$. Ende November war der junge Mann zurack aus Ferrara und traf die Mutter in Lagnieu, bereit, suivant les admonestemens de madame de Nemours sa mere, wie ein Chronist sich ausdrückt, dem König den Treueschwur zu leisten ${ }^{72}$. Anna konnte ihr Glück kaum fassen, schrieb Dankesbriefe an alle Beteiligten und versicherte dem Bruder noch Monate später, Henri sei unsagbar zufrieden mit seinen Ratschlägen, und es verginge keine Stunde, in der er sich dem Onkel nicht zutiefst verbunden fühlte ${ }^{73}$. Der Prinz hatte allen Grund, zufrieden zu sein, denn Heinrich hatte ihm im Vertrag von Folembray vom Januar 1596 großzügige Zugeständnisse gemacht. Eine Generalamnestie für die durch ihn oder seinen Bruder in den Auseinandersetzungen der vergangenen Jahre begangenen Untaten wurde ausgesprochen, Henris Privilegien bestätigt und sein Besitz von allen möglichen Abgaben befreit. Außerdem nahm der König die Teile seines Herzogtums in besonderen Schutz, die an das Herrschaftsgebiet des Herzogs von Savoyen grenzten, und er garantierte Henris Onkel Alfonso seine

\footnotetext{
${ }^{70}$ Anna d'Este an Alfonso d'Este, 11. Okt. 1595, Lyon, in: ASM, Canc. duc., Cart. prin. est. 1458/22. Vgl. die Briefe an Carlo Emanuele di Savoia und Catalina Micaela de Austria vom 1. Okt., in: AST (Cast.), Int., Let. div., Prin. div. 78.

${ }^{11}$ Anna d'Este an Henri de Montmorency, 14. Nov. (1595), Lyon, in: MÜNCH (Hg.), Denkwilrdigkeiten, S. 292. Vgl. die Briefe vom 27. Okt., 12. Nov., 23. Dez. 1595, in: Ibid. S. 260f., BnF, Fr. 3554, fol. 5r, Fr. 3609, fol. 23r.

72 PALMA CAYET, Chronologie novenaire, Bd. 6, S. 249.

${ }^{73}$ Anna d'Este an Alfonso d'Este, 29. Febr. 1596, Lagnieu, in: ASM, Canc. duc., Cart. prin. est. 1458/22. Vgl. die Briefe an Margherita Gonzaga, Alessandro d'Este, Giovan Battista Laderchi und Carlo Emanuele di Savoia vom selben Tag und vom 30. Nov. 1595, in: Ibid. Nr. 1459/23 u. AST (Cast.), Int., Let. div., Prin. div. 78.
} 
in Frankreich gelegenen Besitztümer. Die Summe von 100000 Écus, welche Heinrichs Vorgänger einst Anna zugestanden hatte, sollte zu denselben Konditionen weitergezahlt werden, und sie erhielt daruber hinaus den aus dem Verkauf des im Wald von Montargis geschlagenen Holzes erzielten Gewinn. Daneben wurde ihr und ihrem Sohn fuir die Bezahlung ihrer Schulden ein Aufschub von einem Jahr gewährt ${ }^{74}$.

Angesichts dieser bemerkenswerten Ergebnisse war der Herzog von Nemours seiner Mutter für ihre Unterstützung verbunden und Anna dem Konnetabel für seine Vermittlung. Wăhrend sie diesem in einem Brief ihre Dankbarkeit ausdruckte, redigierte Henri sein Testament und bestimmte die Prinzessin zu seiner Universalerbin. Nicht weniger beeindruckt von diesem Erfolg zeigte sich der Herzog von Savoyen und bat Anna, seinem Agenten bei den Friedensverhandlungen mit Heinrich Unterstützung und Rat zukommen zu las$\operatorname{sen}^{75}$. Trotz allem sollte es noch einige Zeit dauern, bis Henri von dem Vertrag zu profitieren begann, duquel yusques à ceste heure il n'a ancores santi aucun fruit ny commodité, wie es im Herbst hieß, denn der Krieg ging weiter, und noch Ende des folgenden Jahres mußte Anna zugeben: jusques ici quelques ouvertures quil ayt faittes il na peu resevoyr aucune commodite ${ }^{76}$.

\section{2. »Ils vous reconnaissent pour fille de roi $\ll$ Die Lebenssituation der Witwe}

Der Tod ihres Sohnes Charles-Emmanuel, uber den die Prinzessin sagte, er habe sie tant touché au ceur que rien ne me pouvoyt affliger davantage, stellte nur den ersten einer ganzen Reihe von Verlusten dar, die Anna in jenen Jahren

${ }^{74}$ Articles secretz et particuliers des Vertrags zwischen Heinrich IV. und Henri de Savoie, 11. Jan. 1596, in: BnF, Fr. 18709, fol. 586-589; Urkunden Heinrichs IV. fur Anna d'Este und Henri de Savoie, den Verkauf des Holzes betreffend, 11. Jan. 1596, in: AST (Cast.), Int., PGN, cat. 5, m. 6, n. 5, und das Erzbistum Auch betreffend, 2. (nicht 11.!) Mai 1597, in: MÖNCH (Hg.), Denkwirdigkeiten, S. 263f.; Anna d'Este an Carlo Emanuele di Savoia, 29. Febr. 1596, Lagnieu, in: AST (Castello), Int., Let. div., Prin. div. 78; Dies. an Henri de Montmorency, 20. Mărz 1596, Lagnieu, in: BnF, Fr. 3554, fol. 7r. Die Artikel wurden am 31. Mai 1596 vom Pariser Parlament ratifiziert, vgl. SUlly, Les Economies royales, Bd. 2 (1595-1599), hg. v. David BuISSERET, Bernard BARBICHE, Paris 1988, S. 40, Anm. 3.

${ }_{75}$ Anna d'Este an Henri de Montmorency, 14. März (1596), Lagnieu, in: BnF, Fr. 3554, fol. 47r; Testament von Henri de Savoie, 13. Juni 1596, in: AST (Cast.), Int., PGN, cat. 2, m. 13, n. 17; Carlo Emanuele di Savoia an Anna d'Este, 11. Aug. 1596, Rivoli, in: BnF, Fr. 3418, fol. 71r.

${ }_{76}$ Anna d'Este an Henri de Montmorency, 12. Okt. (1596), o.D. (Dez. 1597), in: BnF, Fr. 3554, fol. 16r u. MüNCH (Hg.), Denkwürdigkeiten, S. 290. Vgl. den Brief vom 8. Sept. (1596), in: MC, sér. L, XXIX, fol. 46r. 
zu verkraften hatte. Als sie Ende Mai 1596, nach der Versöhnung ihrer Kinder mit dem König und avec un estrême contantemant du succès de mon voyage in die Hauptstadt zurückkehrte, mußte sie erfahren, daß wenige Wochen zuvor ihre Tochter verstorben war. Im Oktober 1597 ereilte den Bruder in Italien der Tod, und im Februar des folgenden Jahres starb die Schwester Lucrezia. Die Herzogin von Nemours war sechsundsechzig Jahre alt, sie hatte zwei Ehemänner verloren, acht Kinder und vier Geschwister. Zwei Söhne blieben ihr: Charles, der rebellische Herzog von Mayenne, und Henri, der für sein bon naturel bekannte Herzog von Nemours, sowie eine Menge Enkelkinder. Die Aufgaben, welche Anna während der letzten Jahre ihres Lebens zu bewältigen hatte, unterschieden sich nicht sonderlich von denen der vorangegangenen Jahrzehnte. Sie hatte Teil an den gesellschaftlichen Ereignissen der Hauptstadt, wie etwa den Empfängen der ausländischen Botschafter in Paris, sie ubernahm die Verwaltung der Ländereien und Finanzen ihres jüngsten Sohnes, und sie fuhrte wieder einen großen Proze $\beta^{77}$.

\section{Der letzte Prozeß einer streitbaren Fürstin}

Die letzten großen Prozesse der Herzogin von Nemours, ausgefochten vor dem Pariser Parlament, aber auch vor der Rota in Rom, lassen vermuten, daß der regelmäßige Besuch der Gerichtssäle und die Konsultationen mit ihren Advokaten eine Notwendigkeit für sie geworden waren. Die Verfahren verschlangen Geld und fuhrten nicht immer zum Erfolg, vor allem vor der Rota waren die Möglichkeiten für einen Sieg der Prinzessin gering, doch Anna ließ keine Chance ungenutzt. $\mathrm{Zu}$ Hilfe kam ihr die Tatsache, daß sie während ihres langen Lebens am Hof von Frankreich, nach eigenen Worten: pour la longue expérience que j'ay en ceste court, Kenntnisse angehäuft und Kontakte geknüpt hatte, wie sie kaum ein anderes Mitglied des französischen Adels besaß, geschweige denn ein nicht naturalisierter Ausländer ${ }^{78}$. Die Aussicht des Herzogs von Modena auf einen furr ihn vorteilhaften Ausgang des Prozesses gegen seine Cousine, welcher mit dem Tod von Alfonso d'Este im Herbst 1597 begonnen hatte, war daher von Anfang an gering.

Da der Herzog von Ferrara ohne Nachkommen gestorben war, hatte Cesare d'Este, der Sohn von dessen Onkel Don Alfonso, das stark beschnittene Territorium des Fürstentums geerbt und war mit Karren voller Möbel und Kunst-

\footnotetext{
${ }^{77}$ Anna d'Este an Margherita Gonzaga, 1. Juni (1596), Paris, in: ASM, Canc. duc., Cart. prin. est. 1459/23; Dies. an Alfonso d'Este, 11. Okt. 1595, 1. Juni (1596), Lyon, Paris, in: Ibid. Nr. 1458/22. Zu den Empfängen in der venezianischen Botschaft im Juni 1597: RTTER, La Sœur, Bd. 2, S. $193 f$.

${ }^{78}$ Anna d'Este an Alfonso d'Este, 24. Jan. 1587, Paris, in: ASM, Canc. duc., Cart. prin. est. $1458 / 22$.
} 
werke sowie dem gesamten Hof nach Modena gezogen. Hatte er auch auf einen großen Teil seines Erbes in Italien, vor allem auf die Stadt Ferrara verzichten müssen, so erhob der neue Herzog doch zumindest Ansprüche auf Alfonsos französische Besitztümer und auf die Schulden, welche die Krone noch immer bei den Este hatte. Doch Anna, die nicht weniger als ihr Cousin Rechte auf die Hinterlassenschaft des Bruders in Frankreich anmeldete, war ihm zuvorgekommen und hatte von Heinrich IV. die Erlaubnis erhalten, das Erbe mit bénéfice d'inventaire, das heißt unter vorheriger näherer Prüfung antreten zu dürfen, was sie vor der Haftung für eventuell bestehende Schulden bewahrte. So erhielt die Prinzessin bereits erste Zahlungen aus den Einnahmen von Chartres, bevor sie überhaupt mit Cesare und dessen Gemahlin Beileidsschreiben ausgetauscht hatte ${ }^{79}$.

Obwohl die Interessen des Herzogs bei diesem Verfahren nicht nur von seinen Botschaftern, sondern auch vom Bischof von Modena, welcher zu dieser Zeit päpstlicher Nuntius in Frankreich war, vertreten wurden, standen die Chancen für ihn schlecht, nicht zuletzt, weil der König zugunsten der Prinzessin Partei ergriff ${ }^{80}$. Im Mai 1601 verhandelte das Pariser Parlament den Fall. Die Herzogin von Nemours wurde von Antoine Arnauld vertreten, dessen Plädoyer sich auf zwei grundsătzliche Punkte stützte: die Tatsache, daß Cesare Ausländer war und daher keinen Besitz in Frankreich erwerben konnte und den Vorwurf, sein Vater sei illegitimer Sohn gewesen, weswegen er überhaupt keinen Anspruch auf das Erbe seines Cousins erheben könne. In seiner zweistündigen Rede versuchte der Jurist, das Gerechtigkeitsgefühl der Richter anzusprechen und Anna als eine Frau darzustellen, die gemeinsam mit ihren Kindern viel für das Königreich getan habe und sich nun in Gefahr befände, das ihr rechtmäßig Zustehende zugunsten eines Ausländers zu verlieren, welcher den Franzosen noch nie einen Dienst erwiesen habe:

Quant aux services faictz à la France par le demandeur, quels sont-ils pour lesquels il merit[erait] un si particulier privilège? Quoy ces années dernières, où tout le monde ensemble sembloit avoyr conspiré la ruine de ce royaume, où esties-vous pour le sécourir? [...] où est-ce quoy ay vu le duc de Modene [...], quelle esperance mist-on sur ce duc? [...] Bien a-ton veu, Messieurs, quatres enfans de Madame de Nemours employer leur sang pour restaurer ceste monarchie [...]. Seroit cela raison, Messieurs, que les biens de Madame Renée de

\footnotetext{
${ }^{79}$ Erlaubnis Heinrichs IV. für Anna d'Este, das Erbe des Bruders mit bénéfice d'inventaire anzutreten, 29. Nov. 1597, in: AST (Cast.), Int., PGN, cat. 5, m. 5, n. 9; Zahlungsanweisung des Staatsrates für Anna d'Este, 14. Febr. 1598, in: GUÉRIN u.a. (Hg.), Registres des délibérations, Bd. 12, S. 14, Anm. 3; Anna d'Este an Cesare d'Este und Virginia de' Medici, 22. Apr. 1598, Paris, in: ASM, Canc. duc., Cart. prin. est. 1458/22, 1459/23.

${ }^{80}$ Giovan Battista Laderchi an Attilio Ruggieri, 5. Juli 1598, in: Roberto MONTAGNANI, Giovan Battista Laderchi nel governo estense (1572-1618), in: Atti e memorie della Deputazione di storia patria per le antiche provincie modenesi, 10. Ser., 12 (1977) S. 101-153, hier S. 141.
} 
France pussent [...] tumber entre les mains des enfans des courtisannes de Modene, et que Madame de Nemours, fille légitime de Madame Renée de France, en fuse frustrée? ${ }^{81}$

Die rhetorischen Fragen des Advokaten konnten von der Jury nur auf eine einzige Weise beantwortet werden, und nachdem der Staatsanwalt am folgenden Tag sein Plädoyer gehalten hatte, wurde der Prozeß zugunsten Annas entschieden, die bei der Urteilsverkündung persönlich anwesend war ${ }^{82}$.

Viel Geld hatte die Prinzessin dieser Erfolg gekostet. Allein die Gebühren des Anwalts beliefen sich auf sechshundert Écus, und es mußten die huissiers de la grande chambre und celluy qui a faict les coppies en papier entlohnt werden. Zehn Seiten nehmen die frays de yustice in ihrem Rechnungsbuch der ersten Hälfte des Jahres 1601 ein, über tausend Écus sollte sie in jener Zeit für ihre Prozesse bezahlen ${ }^{83}$. Naturlich erhob der Herzog von Modena Einspruch gegen das Urteil, und der König, dem es lieber war, che fra parenti cosi stretti si trattasse d'accordo più tosto che di liti, schlug den Streitparteien einen Kompromiß vor, welcher unter anderem die Heirat des Herzogs von Nemours mit Cesares ältester Tochter vorsah ${ }^{84}$. Nicht zuletzt wegen Annas enormer Forderungen hinsichtlich der Mitgift waren die Hoffnungen auf eine Einigung aber von Anfang an gering, und im Frühsommer 1603 lehnte der Herzog von Modena den Vorschlag definitiv ab mit der Begründung, es mangele nicht an gutem Willen, sondern an der Möglichkeit, eine so hohe Mitgift aufzubringen, vor allem hinsichtlich der anderen Töchter, die er zu versorgen habe - wobei vielleicht auch das Gerucht von Henris angeblicher Impotenz eine Rolle gespielt haben mag $^{85}$. Das Verhältnis sowohl zwischen Anna und ihrer Ferrareser Verwandtschaft als auch zwischen dem Königreich und Modena war jedenfalls auf lange Zeit ruiniert, und in der Folge sollten die Este ihre traditionell frankreichfreundliche Politik aufgeben und sich der spanischen Krone annähern.

Das andere Verfahren, welches Anna seit 1598 an der Rota gegen ihren Cousin unterhielt, trug auch nicht gerade zu einer Verbesserung des Verhältnisses bei. Diesmal ging es um Alfonsos italienische Hinterlassenschaften. Die

\footnotetext{
${ }^{81}$ Antoine ARNAULD, Plädoyer im Prozeß Anna d'Este vs. Cesare d'Este im Protokoll der Gerichtsverhandlung, 15. Mai (1601), in: BnF, Dup. 115, fol. 146-148. Antoine ist nicht zu verwechseln mit seinem Bruder David Arnauld, der in diesem Fall die Gegenpartei vertrat.

${ }^{82}$ Auszug aus dem Register der Entscheidungen des Pariser Parlaments mit dem Plädoyer von Louis Servin, Generaladvokat des Königs, 15. Mai 1601, in: MüNCH (Hg.), Denkwürdigkeiten, S. 368-446. Vgl. L'EsTOILE, Henri IV, Bd. 2, S. 23f. (14. Mai 1601).

${ }^{83}$ Rechnungsbuch 1599-1606, fol. 80-84.

${ }^{84}$ Attilio Ruggieri und ein weiterer unbekannter Verfasser an Giulio Tassoni, 6. Okt. 1601, in: ASF, MP 2935, fol. 331r. Vgl. die Briefe von Heinrich IV. und Henri de Montmorency an Cesare d'Este, 1. Febr. 1603, Paris, Kopien, in: ASM, Casa 339.

${ }^{85}$ Innocenzo del Bufalo an Pietro Aldobrandini, 3. Juni 1603, Paris, in: Correspondance Bufalo, S. 479. Eine Interpretation des Prozesses aus Cesare d'Este freundlich gesinnter Sicht: Alberto GASPARINI, Cesare d'Este e Clemente VIII, Modena 1960, S. 177-183.
} 
Prinzessin gedachte, das Testament ihres Bruders anzufechten und warf Cesare vor, er habe sich der Schätze des letzten Herzogs von Ferrara bemächtigt, darunter Gegenstände aus massivem Gold, die nun in seiner tanière in Modena verborgen lägen ${ }^{86}$. Gleichzeitig stritt sie mit Pietro Aldobrandini um das Erbe von Lucrezia, die der Schwester zwar ihre Anspruche auf das mütterliche Vermögen jenseits der Alpen hinterließ, für ihren italienischen Besitz aber den Kardinal zum Universalerben ernannt hatte, was von Anna anfangs auch anerkannt worden war $^{87}$. Bei diesem Streit ging es unter anderem um den Palazzo der Este in Venedig, welchen der Herzog von Modena an Aldobrandini abgetreten hatte, woraufhin Anna ihren Agenten in der Lagunenstadt beauftragte, sich dem Weiterverkauf des Gebäudes entgegenzustellen ${ }^{88}$. In Rom wurde sie von Antoine Favre vertreten, einem der Präsidenten von Genevois. Gemeinsam mit anderen Beratern der Prinzessin reiste er in jenen Jahren wiederholt in die Ewige Stadt, und seine Auftraggeberin lobte, er habe tellemant éclercy mes droys et prétantions par sa prudance et sage conduitte que ye puis dire sans luy mes affaires fussent demeurées an ténèbres. Doch trotz des großen, auch finanziellen Aufwandes - Favre und seine Kollegen hatten in Rom vierhundert Écus Schulden hinterlassen - verlor die Prinzessin den Prozeß vor der Rota ${ }^{89}$. Nach seiner Rückkehr nach Paris verhandelte Favre lange mit den Vertretern des Kardinals, um wenigstens in diesem Fall zu einer Einigung zu gelangen, aber hinsichtlich des Palastes in Venedig fand Anna sich bald con le mani piene di vento, und noch im Oktober 1602 beschwerte sich der Nuntius in Frankreich uber ihre Winkelzüge und ihr schlechtes Benehmen ${ }^{90}$.

${ }^{86}$ ARNAULD, Plädoyer, fol. 147v. Zu Annas Ansprüchen in Italien: Madama di Nemours pretende la sussessione universale de l'ultimo duca di Ferrara suo fratello quanto alli beni da luy lasciati in Italia, o.D. (ca. 1600), in: ASM, Casa 339.

${ }^{87}$ Verzichterklärung von Anna d'Este bezilglich der Ansprüche, die sie auf den Pietro Aldobrandini zugedachten Anteil an Lucrezias Erbe erheben könnte, 14. Apr. 1598, ratifiziert am 25. Juni 1599, in: ASM, Casa 337; Testament von Lucrezia d'Este, 4. Febr. 1598, in: ASF, DU, cl. III, 3, fol. 262-265.

${ }^{88} \mathrm{Vgl}$. zahlreiche diesen Streit betreffende Briefe des Nuntius in Frankreich an den Kardinal Aldobrandini, März-Okt. 1602, in: Correspondance Bufalo, S. 261-363. Bei dem venezianischen Palazzo der Este handelt es sich um den Fondaco dei Turchi, heute Sitz des Museums furr Naturgeschichte.

${ }^{89}$ Anna d'Este an Carlo Emanuele di Savoia, 6. Sept. (1599), Paris, in: AST (Cast.), Int., Let. div., Prin. div. 78; Bezahlung einer diesbezlglichen Rechnung vom 25. März 1599 im Ausgabenbuch des Jahres 1602, in: AST (Cast.), Int., PGN, cat. 3, m. 1, n. 1; Wechselschein über 400 Écus, 22. März 1602, in: Correspondance Bufalo, S. 29; Zusammenstellung verschiedener, das Urteil der Rota betreffender Dokumente, 14. Jan. 1602, in: Informationes Facti et Juris, in causa Ferrarien. bonorum, iterum proponenda ex eisdem. Pro Illustriss. et excellent. D. Anna Estensi Namurci et Camutum Ducissa: Contra Sereniss. D. Caesarem Estensem Ducem Mutinae: Romae, in Rota sacri Palatij Apostolici, o.O. o.J. [1602].

${ }^{90}$ Vincenzo Ungarino und Innocenzo del Bufalo an Pietro Aldobrandini, 2. Juli, 19. Aug., 18. Okt. 1602, in: Correspondance Bufalo, S. 312, 338, 363. 


\section{Die salte Damer des Hofes}

Im November 1592, als Anna in Paris noch »Königinmutter« genannt wurde und ihre Versöhnung mit dem König nicht abzusehen war, soll einer von dessen Sekretären ihr bei einem Besuch in der Hauptstadt bezüglich des Ansehens, welches sie in Heinrichs Umfeld genoß, gesagt haben: on n'ignore point de par delà vos qualités, vos mérites et vos grades: ils vous reconnaissent pour fille de roi $i^{91}$. Nicht einmal die schwerstwiegenden politischen Unstimmigkeiten konnten den Tatbestand schmälern, daB in den Adern der Herzogin das Blut eines franzősischen Königs floß. Anna selbst war sich ihres Wertes bewußt und bestand auf die entsprechende Behandlung, wie etwa anlaßlich der Taufe einer natürlichen Tochter Heinrichs IV. im Dezember 1596, an der die Prinzessin nicht teilnehmen wollte, da sie sich mit einer anderen Dame nicht uber die Rangfolge hatte einigen können ${ }^{92}$. So war es nur natürlich, daß die Herzogin von Nemours von Heinrich dazu auserkoren wurde, seine Braut bei ihrer Ankunft in Frankreich zu empfangen und die Oberaufsicht uber deren Hof zu übernehmen, ed essendo princepessa di tanta noblità e di tanta bontà come veramente ella è tenuta da ciascuno, è stata lodatissima questa elettione, et la regina ne doverà sentire gran contento, anche per essere Italiana ${ }^{93}$. Anna war eine gute $>$ Kompromißkandidatin<, nicht nur, weil sie Italienerin war, aber schon lange in Frankreich lebte, sondern auch weil sie, als einstige Anhängerin der Ligue und spătere Verteidigerin der Thronfolge durch den Navarresen, den schwierigen Weg Heinrichs zur Krone versinnbildlichte.

Als Maria von Medici Anfang November 1600 mit einer Flotte von siebzehn Galeeren in Marseille anlangte, war die Herzogin von Nemours die erste Dame, welche Heinrichs Braut am Kai willkommen hieß. Bis zu ihrer Ankunft in der Hauptstadt mehrere Wochen später sollte sie der neuen Königin nicht mehr von der Seite weichen, so daß es nicht verwunderlich ist, in den Quellen wiederholt die Formulierung »Madame de Nemours et autres dames« zu finden. Derart beschäftigt war Anna in jenen Tagen, daß sie nicht einmal die Zeit fand, sich um ihre eigenen dringenden Angelegenheiten zu kümmern: $j$ 'ay à touttes heures tant d'occupation et autres anpeschemens pour le service de la Royne, que cette commodité m'en est du tout ostée ${ }^{94}$. Von Marseille aus bega-

${ }^{91}$ L'ESTOILE, Journal Henri IV, Bd. 1, S. 193 (3. Nov. 1592).

${ }^{92}$ Claude GroularD, Premier Président du Parlement de Normandie, Mémoires ou Voyages par lui faits en Cour (1588-1604), hg. v. Claude-Bernard PETITOT, Paris 1826, S. $342 f$.

${ }_{93}$ Baccio Giovannini an Ferdinando de' Medici, 3. Mai 1600, Paris, in: ASF, MP 4615, fol. 284v.

${ }^{94}$ Beschreibung der Ankunft Marias von Medici in Marseille, 3. Nov. 1600, in: Discours de ce qui s'est faict et passé au voiage que le Roy et Messieurs de son conseil ont faict en 1600 au mois de Juin tant en la ville de Lion qu'en celle de Marseille, o.D. (Anfang Nov. 1600), Kopie des 17. Jh., in: BnF, Fr. 4020, fol. 36-48, hier fol. 42f. Filippo Molza an Cesare d'Este, 31. Okt. 1600, Chambéry, in: ASM, Canc. duc., Amb. Francia 101: erwähnt Anna 
ben sich die Damen nach Lyon, wo im Dezember der König erwartet wurde, und die Chronisten erzählen, nach dessen Ankunft habe die Herzogin beim Aushandeln der ersten Liebesnacht des Paares als Botin fungiert ${ }^{95}$. Bei der Hochzeitszeremonie trug die Prinzessin Marias Schleppe und saß beim anschließenden Festbankett an ihrer Seite, und als die Geburt des Thronfolgers erwartet wurde, begleitete sie die junge Königin nach Fontainebleau und leistete an ihrem Bett Beistand bei der Niederkunft ${ }^{96}$. Ab Anfang des Jahres 1601 ist die Herzogin von Nemours als superintendante de la maison im Hofstaat der Königin vermerkt. Ein Vergleich mit den Haushalten von Marias Vorgăngerinnen zeigt, daß sich die Zusammensetzung des Gefolges vollkommen verändert hat, und daß Anna die einzige salte` unter all den sneuen` Damen ist, deren Namen im Hofstaat der Gemahlin Heinrichs III. und der Königinnen vor ihr nicht auftauchen ${ }^{97}$.

Die Prinzessin war nun fast siebzig Jahre alt und hatte die Oberaufsicht über den Hof der Königin inne, wofür sie mit einer Pension von tausendzweihundert Livres entschädigt wurde, während sie zuvor stets zu den mit achthundert Livres vergüteten dames gezählt hatte ${ }^{98}$. Noch Ende der 1590er Jahre hatte sie sich selbst als alt und presque inutille bezeichnet, nun fuhrte sie Schutzbefohlene bei der Königin ein und schrieb Empfehlungen an die Gouvernante von Marias Kindern für diejenigen, welche den Dauphin zu sehen nach Paris rei$\operatorname{sten}^{99}$. In Annas Rechnungsbüchern jener Zeit findet sich eine Liste derjenigen bei Hofe, die von ihr mit Geldgeschenken bedacht wurden, was beweist, daß sie wie eh und je in den königlichen Schlössern verkehrte. Darunter die fourriers du corps des Königs und der Königin, die huissiers de chambre de la Royne sowie die königlichen Lakaien, Portiere und Kutscher, außerdem die Wachen der Herren Villeroy, Bellièvre und Rosny sowie die huissiers du

con moltissime altre signore. Philippe HURAULT, Abbé de Pontlevoy, Eveque de Chartres, Mémoires, hg. v. Claude-Bernard PETITOT, Paris 1823, S. 483: madame de Nemours et autres. Anna d'Este an die Mitglieder der Rechnungskammer von Genevois, 14. Dez. 1600, Lyon, in: AST (Cast.), Int., PGN, cat. 2, m. 14, n. 3.

${ }_{95}$ HuRAUlT, Mémoires, S. 483f.; L'EsTolle, Journal Henri IV, Bd. 1, S. 636 (9. Dez. 1600).

${ }^{96}$ Cérémonie des nopces du Roy Henry quatriesme en l'an mil six cens, (Mitte Dez.) 1600 , Kopie des 17. Jh., in: BnF, Fr. 4321, fol. 41-43, hier fol. 42r. Zu Annas Anwesenheit bei der Geburt des späteren Ludwig XIII.: RITTER, La Sœur, Bd. 2, S. 398-400.

${ }^{97}$ Hofstaat der Königin Maria von Medici, o.D., in: BnF, NAF 9175, fol. 413-424, hier fol. 413r; Hofstaat der Königinnen Elisabeth von Österreich und Luise von Lothringen, o.D., in: Ibid. fol. 399-412; Hofstaat der Königin Maria Stuart, o.D., in: PARIS (Hg.), Négociations, S. 744-750; Hofstaat der Königin Katharina von Medici, o.D., in: MÉDICIS, Lettres, Bd. 10, S. 504f.

${ }_{98}$ Quittung von Anna d'Este über 200 1.t., ein Viertel ihrer jählichen Pension, 6. Apr. 1559, in: AN, K 92, Nr. 21.

${ }^{99}$ HURAULT, Mémoires, S. 484; Anna d'Este an Françoise de Longuejoue, o.D. (Anfang 17. Jh.), in: BnF, Fr. 3649, fol. 49r; Dies. an Henri de Montmorency, 6. Okt. (1598), in: BnF, Fr. 3574, fol. 40r. Das Zitat : Dies. an Carlo Emanuele di Savoia, 17. Sept. 1599, in: AST (Cast.), Int., Let. div., Prin. div. 78. 
conseil; sie beschenkte aber auch die nains de la Royne und die petitte folle de Madame, saur du Roy ${ }^{100}$. In den letzten Jahren ihres Lebens scheint Anna von den Fürstinnen und Fürsten Europas als die große, alte Dame Frankreichs angesehen worden $\mathrm{zu}$ sein, man wunschte ihr prosperita, et consolatione, sie genoß estime und affection und war stets bestens über alle Neuigkeiten informiert ${ }^{101}$. Wenn sie bei Festen am Hofe an der Tafel des Königs und der Königin speiste, wurde sie direkt neben dem Herrscherpaar plaziert, und noch im Oktober 1602 begleitete sie Maria zu der prächtigen Messe in Notre-Dame, mit welcher das Bündnis mit den Schweizern gefeiert wurde. Es scheint dies das letzte Mal gewesen zu sein, daß die Herzogin sich bei einem großen zeremoniellen Ereignis sehen ließ, bei der Taufe des Dauphin und seiner Schwestern vier Jahre später war sie nicht anwesend ${ }^{102}$.

\section{Haus und Haushalt: Fürstliches Leben im Hôtel de Nemours}

Von ihrer letzten großen Reise im Herbst und Winter 1600 abgesehen, verließ Anna nach der Rückkehr im Anschluß an die Versöhnung ihrer Kinder mit dem König die Hauptstadt nur noch selten. Die letzten zehn Jahre ihres Lebens verbrachte sie im Hôtel de Nemours, welches sich links der Seine, nahe dem Pont Saint-Michel befand, zwischen der Kirche Saint-André-des-Arts und dem Kloster der Augustiner, die es beide nicht mehr gibt. Zu dem Gebäudekomplex mit Fassade und Eingang zur Rue Pavée, heute Rue Séguier, gehörten neben dem Wohnhaus der Herzogin und seinem Innenhof einige Wirtschaftsgebäude und ein großer Garten. Das Grundstück erstreckte sich damals noch uber die gesamte Länge der Rue Pavée; die heutige Rue de Savoie, welche den ehemaligen Garten der Herzöge von Nemours durchkreuzt, stammt aus dem 17. Jahrhundert (Abb. 9). Gleich nach Ende der Belagerung der Hauptstadt hatte Anna mit umfassenden Renovierungsarbeiten an und in den Gebäuden

\footnotetext{
${ }^{100}$ Rechnungsbuch 1599-1606.

${ }^{101}$ Marino Grimani, Doge von Venedig, an Anna d'Este, 22. Aug. 1605, Venedig, in: MüNCH (Hg.), Denkwürdigkeiten, S. 259f.; Carlo Emanuele di Savoia an dies., 24. Mai 1604, Turin, in: BnF, Fr. 3647, fol. 53r; Eleonora de' Medici, Herzogin von Mantua, an dies., 16. März 1606, Mantua, in: Ibid. fol. 76r; Henri de Savoie an Leo XI., 16. Apr. 1605, Paris, in: Ibid. fol. 72r; Jacques Davy du Perron, Bischof von Evreux, und der Kardinal von Peronne an dies., (27. Juni) 1604, Condé, in: MÜNCH (Hg.), Erinnerungen, Bd. 2, S. 121f.; Diego de Lizárraga an Philipp III., 18. Aug. 1606, Paris, in: AN, 21 Mi 63.

${ }^{102}$ Beschreibungen der Taufen, 13.-14. Sept. 1606, in: GODEFrOY (Hg.), Cérémonial Francois, S. 169-192 u. BnF, Fr. 4324, fol. 28f., Fr. 4325, fol. 32vf., Dup. 931, fol. 161f.; Beschreibung der Festmesse, 20. Okt. 1602, in: GUÉRIN u.a. (Hg.), Registres des délibérations, Bd. 13, S. 37. Zur Plazierung an der königlichen Tafel: Louise de Coligny an Charlotte Brabantina von Oranien-Nassau, 27. Aug. 1601, Paris, in: Louise de COLIGNY, Princesse d'Orange (1555-1620), Correspondance, hg. v. Paul MARCHEGAY, Léon MARLET, Paris 1887 , ND 1970, S. $192 \mathrm{f}$.
} 
begonnen. Sie ließ ihre privaten Räume erneuern, zu denen, nicht anders als in den Häusern anderer Fürstinnen, neben dem Schlafzimmer mit seiner antichambre ein Büro und ein Kabinett sowie weitere kleinere Răume gehơrten, außerdem die Gemächer ihrer Söhne und den großen Saal. Maurer, Schreiner, Dachdecker, Schlosser, Flaschner, Plattenleger, Glaser und Maler waren ununterbrochen damit beschäftigt, Dächer, Türen und Fenster zu erneuern, Wände zu vertäfeln und Parkett zu legen. Die Prinzessin kaufte Vorhänge für ihr Schlafzimmer und Tapisserien aus versilbertem und vergoldetem Leder, mit denen die Wande bespannt wurden und die sie mit ihrem Monogram versehen lie $\beta^{103}$.

Die Einrichtung von Annas Privatgemächern war ganz in schwarz gehalten: ein Himmelbett aus schwarzem Samt mit Vorhängen aus schwarzem Damast, schwarz bezogene Stühle, an den Wänden Tapeten aus schwarzem, mit Silberbesatz versehenem Leder. Ihr Schlafzimmer, von dem aus man in den Hof blickte, war geschmückt mit den Portrăts der Söhne, von denen einige erst kürzlich angefertigt worden waren. So gab die Prinzessin im April 1593 zwölf Écus für ein Bild von Charles-Emmanuel aus, bestellte bei einem Maler la peinture de feu mon pauvre fils le cardinal und bezahlte wiederholt einen gewissen mestre Yerosme für seine Gemălde. Der Mode der Zeit entsprechend waren mehr als fünfzig weitere Porträts in der Galerie zu sehen, ähnlich der Sammlung, die im Hôtel der Katharina von Medici hing. Unter den Portrăts befanden sich Bildnisse von Annas Eltern, den beiden Ehemännern und der Tochter, außerdem von Fürstinnen und Fürsten, die sie kannte oder gekannt hatte: Katharina von Medici und ihre Töchter, Maria von Medici und ihr Sohn, die Herzöge von Savoyen, eine der spanischen Infantinnen sowie die Könige Frankreichs von Franz I. bis Heinrich IV., alle - außer Heinrich III., dem Mörder ihrer Kinder ${ }^{104}$.

Neben den Gemälden hatte die Herzogin eine Sammlung verschiedener großer und kleiner Standuhren zusammengetragen, darunter une grande horloge carré sonnante à mettre sur table, avecq son reveille matin, le tour de la boiste de pieces sizellées en relief. Die Begeisterung der Prinzessin für ihre Uhren ist an den fur deren Reparatur getătigten Ausgaben abzulesen, die nicht selten den Wert der Stücke überstiegen ${ }^{105}$. Auch war sie bereit gewesen, einen in Gold gefaßten Smaragd im Wert von fünfhundert Scudi, den der Vater ihr hinterlassen hatte, damit sie ihn zu seinem Gedenken am Finger trüge, gegen eine große, die Bewegung der Planeten anzeigende Uhr aus dem Nachlaß der

${ }^{103}$ Rechnungsbücher 1591, 1593-II.

${ }^{104}$ Anhang A, fol. 5r-v, 6r; Rechnungsbuicher 1591, fol. 19r (Mai), 1593-II (Jan., Apr.). Bei dem mit »l'infante d'Espagne« bezeichneten Porträt handelt es sich wohl um eine Darstellung von Isabel Clara Eugenia de Austria. Zu den Bildern im Hôtel de la Reine: MICHAHELLES, Inventory, S. 6f., 30-33.

${ }^{105}$ Rechnungsbüicher 1591, fol. 4r, 29r, 31r, 35r (Mărz, Juli, Aug., Okt.), 1593-II (Apr.). Das Zitat: Anhang A, fol. 12vf. 
Mutter einzutauschen ${ }^{106}$. Nach Annas Tod nahmen ihre demoiselles vier Stilkke der Sammlung an sich, darunter eines mit goldenem Gehäuse, während sich das wertvollste Exemplar in einem der Schlösser in Savoyen befunden haben dürfte: eine mit Rubinen und Smaragden besetzte Kristalluhr, con la coppola d'oro, et la sommità con una picola figura di smeraldo ${ }^{107}$. Ein Vergleich mit anderen Fürstenbehausungen des 16. Jahrhunderts zeigt, daß der Geschmack der Prinzessin bei der Einrichtung ihrer Wohnung der Mode der Zeit entsprach. So war die Ausstattung des Hôtel in der Rue des Deux-Écus unter Katharina von Medici ebenso in schwarz gehalten wie Annas private Gemächer, und zu Zeiten, als Catherine de Bourbon das Gebăude bewohnte, schmückte eine Sammlung von Bildern das Foyer. Das Inventar des Schlosses von Thouars, in dem Gabrielle de Bourbon starb, fuhrt vergleichbare Posten auf wie das des Hauses der Herzogin von Nemours, während an den Wänden des Hôtel de Montmorency ăhnliche Portrăts europäischer Fürsten hingen wie an denen der Galerie in der Rue Pavée ${ }^{108}$.

Aufgewartet wurde der Herzogin von ihrem Gefolge, welches Anfang der 1590er Jahre rund funfzig Personen umfaßte, zehn davon weiblich, im Laufe der Zeit aber schrumpfen sollte und 1606 aus nur noch dreißig Männern und sechs Frauen bestand, von denen etwa dreißig Prozent italienischer Herkunft waren. Einige von ihnen waren die ganzen Jahre über im Hôtel de Nemours anzutreffen, wie Isabelle de Fresne und Ippolita Sacca, letztere eine Italienerin und beide in Annas chambre tătig, ebenso ihre männlichen Kollegen Jourdan Marquet und Jean Breton, welcher Le Prince genannt wurde ${ }^{109}$. Daneben fin-

${ }^{106}$ Testament von Ercole d'Este, 13. März 1558, in: Carlo ZAGHI, Saggio di bibliografia di Renata di Francia e della riforma in Ferrara, in: Atti e memorie della Deputazione ferrarese di storia patria 28(1931) S. 17-130, hier S. 118; Giulio Alvarotti an Alfonso d'Este, 19. Apr. 1560, Amboise, in: ASM, Canc. duc., Amb. Francia 36, fasc. II, fol. 7v; Übereinkunft zwischen Anna d'Este und Jacques de Savoie auf der einen sowie Alessandro Fiaschi auf der anderen Seite, den beweglichen Besitz von Renée de France betreffend, 9. Juli 1575, in: ASM, Casa 333, S. 2; Ratifikation des Vertrags zwischen Anna und Alfonso d'Este, das matterliche Erbe betreffend, 8. Sept. 1583, in: Ibid. Nr. 334, S. 3.

${ }_{107}$ Die Uhr ist verzeichnet in Annas gabinetto im SchloB von Lingotto: Post-mortem Jacques, fol. 71r. Vgl. Anhang A, fol. 22v.

${ }^{108}$ Inventar des Schlosses von Thouars, 1516, in: Philippe CONTAMINE, Espaces féminins, espaces masculins dans quelques demeures aristocratiques françaises, $\mathrm{XIV}^{e}-\mathrm{XVI} I^{e}$ siècle, in: Jan Hirschbiegel, Werner Paravicini (Hg.), Das Frauenzimmer. Die Frau bei Hofe in Spätmittelalter und fruher Neuzeit, Stuttgart 2000, S. 79-90, hier S. 86; Inventar des Hôtel de Montmorency, 1556, in: MIROT, Montmorency, Bd. 79, S. 383-385, 412f. Zu Katharina von Medici: BonNAFFé, Inventaire des meubles, S. 242f. Zu Catherine de Bourbon: EURICH, Power, S. 189.

${ }^{109}$ Tägliche Ausgaben für Essen und Trinken von Anna d'Este und ihrem Gefolge, 1592, in: AST (Piave), ACP, Art. 806, par. 2, reg. 170; Rechnungsbuch 1593-I von Anna d'Este über die ordentlichen Ausgaben, in: Ibid. reg. 171; Rechnungsbuch 1601-1607 von Anna d'Este, in: Ibid. reg. 172; Rechnungsbuch 1599-1606; Anhang A. Die bei MAJOR, Noble Income, S. 39, aufgeführten Haushalte von Fürstinnen bestanden zu 16-25\% aus Frauen, ein Anteil 
den sich immer auch einige außerordentliche Posten im Haushalt verzeichnet, die petite Anne und der petit Jacques etwa, bei denen es sich wahrscheinlich um Kinder von Bediensteten handelte, die nicht nur gespeist und gekleidet, sondern gemeinsam mit einem der Zimmermädchen auch in Lesen und Schreiben unterrichtet wurden: au pettit Yaques, à Madelayne et à la pettitte Anne quarante sous pour leur metresse d'escolle à lire et écryrre. So setzte Anna die Tradition der Mutter fort, für die Kinder des Haushaltes eine eigene kleine scuola zu unterhalten ${ }^{110}$.

Nicht nur die Kinder, alle Mitglieder der maison wurden von der Herzogin gekleidet. Die Pagen und Lakaien, der Kutscher und der Maultiertreiber sowie der Schweizer, welcher das Tor bewachte, erhielten zweimal im Jahr neue Kleidung, die aus drei Hemden aus weißem Tuch, schwarzen Beinkleidern und einem Mantel de bon drap noir bordé de pasement noir garny de boutons à longue queue et le colet double de velours bestand, sowie aus Schuhen, einem Hut und einem Gürtel ${ }^{111}$. Natürlich wurden die Bediensteten auch von ihrer Auftraggeberin gespeist, so daß im Hôtel de Nemours täglich ein ganzer Schinken und ein halbes Schaf, drei Rinderzungen und ein Kalbsmagen, Enten, Tauben und anderes Geflügel, oder aber Karpfen, Forellen, Hechte, Barsche, Aale und Muscheln verbraucht wurden, dazu Äpfel und Birnen, Kirschen, Aprikosen und Orangen, Mandeln und Nüsse sowie Zucker und Kalbsfußße für Gelee. Hinzu kamen Brot, Wein, Öl und Salz, außerdem verschiedene Gewürze, Zwiebeln und Petersilie, Essig, Senf und Safran. Vierzig bis fünfzig Livres täglich kostete Anna Anfang der 1590er Jahre die Versorgung ihres Gefolges mit Lebensmitteln, und im Juli 1603 kaufte sie Vorräte für zwei Jahre im Wert von sechstausend Livres. Im Winter mußten außerdem die Kamine mit Holz versorgt werden; in ihrem Rechnungsbuch zählte die Prinzessin über siebzehn große Scheite, welche an die in ihrem Hôtel Lebenden verteilt wurden ${ }^{112}$. Nicht alle Mitglieder des Haushaltes wohnten mit ihrer

von $20 \%$ weiblicher Mitglieder stellt also keine Besonderheit dar. Vgl. Sharon KETTERING, The Patronage Power of Early Modern French Noblewomen (1989), in: DIES., Patronage in Sixteenth- and Seventeenth-Century France, Aldershot, Burlington 2002, Art. V, S. 826. Von den 20 in DuBost, France italienne, S. 361, genannten Angehörigen von Annas maison waren 14 Franzosen, was einem Prozentstatz von $70 \%$ entspricht. Ob es sich bei Ippolita Sacca um die in den Rechnungsbuchem 1535, fol. 13v, 1540, fol. 15v, 81r, 1544, fol. 17r als demoiselle oder fille gefuhrte Ypolite Socque handelt, ist unklar.

${ }^{110}$ Rechnungsbuch 1593-II (Aug.). Die Lehrerin verdiente einen halben Écu monatlich. Vgl. auch die den Kauf von Kleidung für die Kinder betreffenden Einträge in: Ibid., sowie FRANCESCHINI, La corte, Kap. 4.

${ }^{111}$ Vertrag über den Kauf von Kleidungsstücken zwischen Anna d'Este und Jean Berault, 20. Febr. 1602, in: AN, m.c. VIII, 559, fol. $188 f$.

112 Verträge über den Kauf von Lebensmitteln zwischen Anna d'Este und Ozian Begny, 1. Juli 1603, 1. H. 1606, in: AN, m.c. VIII, 561, fol. 493-498, 568, fol. 67-73; Rechnungsbücher 1569, 1591 (Nov.), 1593-I (16. Nov.). Einer dieser Verträge über die Versorgung des Hofes von Anna und Jacques für mehrere Jahre mit Lebensmitteln in: LELOUP, Anne d'Este, 
Brotgeberin unter einem Dach, die meisten wurden in gemieteten Gebäuden untergebracht, beispielsweise in einem Haus des prévôt von Paris, welches Anna im Herbst $1591 \mathrm{zu}$ diesem Zwecke hatte renovieren und reinigen las$\operatorname{sen}^{113}$. Weitere Ausgaben hatte die Herzogin furr ihre Pferde und Maultiere zu tätigen, denn dreißig Tiere standen in ihren Ställen und fraßen jeden Tag rund achtzig Bündel Heu, die Pferde mußten beschlagen und die Wagen sowie die mit schwarzem Samt und Satin bezogene Sänfte instand gehalten werden ${ }^{114}$.

Die Sorge der Prinzessin um die Mitglieder ihres Gefolges ist an den zu deren Gunsten getätigten Schenkungen abzulesen. Da seit Beginn der Religionskriege kaum noch ein Anstieg der Löhne verzeichnet werden konnte, waren vor allem Bedienstete in niedrigeren Positionen auf diese Extrazuwendungen angewiesen, welche Unterkunft und Verpflegung, Kleidung und medizinische Versorgung ebenso einschlossen wie einmalige Geldgeschenke, Pensionen, Mitgiften und Hinterlassenschaften. So überstieg der Wert der von Catherine de Bourbon, Schwester Heinrichs IV., den Mitgliedern ihrer maison gewährten Geschenke spätestens seit 1594 die ordentlichen Löhne deutlich. Die Damen de Panjas und de La Barre beispielsweise erhielten im Jahre 1598 einen Lohn von je tausendzweihundert Livres, der durch Extrazuwendungen in Höhe von rund siebentausend Livres pro Person aufgestockt wurde. Und während das Einkommen von Marguerite de Thignonville, die erst für Jeanne d'Albret, später für deren Tochter tätig war, zwischen 1572 und 1591 nicht mehr stieg, empfing sie nach dem Tod der Königin von Navarra innerhalb von sechs Jahren 30000 Livres als Geschenk ${ }^{115}$.

Ähnliche Verhältnisse können auch für den Haushalt der Herzogin von Nemours angenommen werden. $\mathrm{Zu}$ den von Anna gewährten Extrazuwendungen zählten kleine Gaben zu besonderen Anlässen, wie etwa pour le basteme du consierge sis écus oder à la fille de Gilles quatre écus pour présant à ses nozzes, ebenso wie die Unterstützung bei Krankheit oder nach Unfällen: quatre écus à Yourdan pour lui ayder an sa malladie oder à Darnassal pour la fayre panser quarante sou ${ }^{116}$. Einige der demoiselles erhielten sehr hohe

S. 57-59. Vgl. den Vertrag zwischen Marie de Bourbon und ihren Lebensmittelhändlern von 1561, in: E. SARRIAU, Traité passé en 1561 par Marie de Bourbon, duchesse de Nivernais, avec les fournisseurs de sa maison, in: Bulletin de la Société nivernaise des lettres, sciences et arts, 3. Ser., 5 (1893) S. 137-144.

${ }^{113}$ Rechnungsbuch 1591, fol. 33r (Sept.), 34v (Okt.).

${ }^{114}$ Rechnungsbücher 1593-I (16. Nov.), 1591, fol. 2r, 16r, 17r, 25r, 41v (Febr.-Dez.), 1593II (März); Vertrag zwischen Anna d'Este und Ozian Begny über den Kauf von Pferdefutter, Okt. 1603, in: AN, m.c. VIII, 562, fol. 262f. Zur Sänfte: Anhang A, fol. 21 r.

${ }^{115}$ EURICH, Power, S. 113, 120, 122. Vgl. Mack P. HolT, Patterns of Clientèle and Economic Opportunity at Court during the Wars of Religion: The Household of François, Duke of Anjou, in: French Historical Studies 13 (1984) S. 305-322, hier S. 318f.; KETTERING, Household Service, S. 73-75.

${ }^{116}$ Rechnungsbücher 1591, fol. 8r (Marz), 1593-II (Mai, Juni, Sept.). Vgl. Rechnungsbuch 1599-1606, fol. 87r-91v (Jan.-Juni 1601): À Jehan Baptiste de la cuisine que Madite dame 
Summen, beispielsweise Ippolita Sacca, die nach vielen Dienstjahren viertausendflinfhundert Livres übereignet bekam, oder Anne du Fresne, der die Herzogin und ihr Sohn gar zweitausend Écus schenkten. In diesem Fall war das Geld als Mitgift gedacht und wurde erst dreieinhalb Jahre später ausgezahlt, kurz vor der Hochzeit der jungen Frau mit einem von Annas Sekretären. Es war dies nicht die einzige Heirat, welche zu bezeugen die Herzogin gebeten wurde, ihre Unterschrift findet sich auch unter weiteren Eheverträgen, etwa dem ihrer Kammerzofe Anne Bellando, die sich mit einem Kammerdiener des Herzogs von Nemours verband ${ }^{117}$. Die größte Sorgfalt verwendete die Prinzessin jedoch auf das Verhältnis zu ihren Beratern und Sekretären, vor allem zu Guillaume Le Cirier, Seigneur de Neufchelles, und François de Sacquespée, Seigneur de Sélincourt und Kommendatarabt von Saint-Evroult. Die Herren bewohnten mehrere Răume im Hôtel de Nemours, die mit ihren eigenen Möbeln und Büchern eingerichtet waren. Über die Jahre hinweg hatte Anna wiederholt Schenkungen ihnen zugunsten getătigt und sie zu ihren Testamentsvollstreckern eingesetzt mit der Begründung, die beiden leisteten ihr seit über dreißig Jahren treue und gute Dienste ${ }^{118}$.

\section{Verwaltungstätigkeit und finanzielle Situation}

Als Charles-Emmanuel im Sommer 1595 starb, hatte der Marquis von SaintSorlin sein füfundzwanzigstes Lebensjahr noch nicht erreicht und war somit minderjährig. Die Herzogin von Nemours ubernahm daher die Verwaltung des Herzogtums, beschwerte sich in Turin in seinem Namen über yans de guerre, contributions et levées de deniers und bat um Beseitigung der Mißstände, afin de soulager ce pauvre peuple tant affligé et ruyné, qui autremant ne pourra

luy donne pour aider à marier sa fille 15 écus und Aux filles de Madite dame pour aller à la nopce de ladite fille de Jean Baptiste en don 2 écus.

${ }_{117}$ Oberschreibung von 4500 l.t. von Anna d'Este an Ippolita Sacca, 18. Mărz 1604, in: AN, m.c. VIII, 563, fol. 141r; Schenkung von 2000 Écus von Anna d'Este und Henri de Savoie an Anne du Fresne, 20. Aug. 1598, in: AN, Y 141, fol. 111r; Auszahlung des geschenkten Geldes, 20. Febr. 1602, in: AN, m.c. VIII, 559, fol. 187r; Heiratsvertrag zwischen Anne du Fresne und Jean Gaignant, 1. Mai 1602, in: Ibid. fol. 350f.; Heiratsvertrag zwischen Anne Bellando und François de Varenne, 2. Mai 1607, in: Ibid. Nr. 570, fol. 337-339. Vgl. die Schenkung von 3000 1.t. an Anne Darnassal, 21. Sept. 1605, in: Ibid. Nr. 567, fol. 200r, und den Vertrag der Heirat von Anne de Bonmercat, Annas fille d'honneur, mit dem zum Gefolge des Herzogs von Nemours gehörenden Philippe de Fontaine, 14. Mai 1601, in: AN, Y 140 , fol. 253r.

118 Schenkungen von Anna d'Este an Guillaume Le Cirier und François de Sacquespée, 30. Dez. 1570, 1. Sept. 1583, 6. Mărz 1584, in: AN, Y 111, fol. 150v, Y 125, fol. 363r u. Anhang A, fol. 21v, 22r, Testament von Anna d'Este, Entwurf, o.D., Kopie des 17. Jh., in: BnF, 500 Colb. 81, Nr. 191ter (fol. 98v). 
payer ce qu'il doyt à mondit fils ${ }^{119}$. Auch Jahre später noch besaß Anna eine Generalvollmacht ihres Sohnes, öffnete seine Post und kümmerte sich um die Geschăfte, wie an dem Memorandum zu erkennen ist, in welchem sie dem Herzog von Savoyen die ynfinité de pertes et dommages darlegte, die Henri in den Ländereien seiner Apanage hinzunehmen habe. Sechs Forderungen beinhaltete das Papier, und Carlo Emanuele kam ihnen in allen Punkten entgegen $^{120}$.

Das Verhältnis des Herzogs von Nemours zu seiner Mutter ist ein gutes Beispiel furr die Bedeutung adliger Damen für die Verwaltung von Familienbesitz: Wäre Henri zu diesem Zeitpunkt verheiratet gewesen, hătte die Gemahlin die Geschäfte gefuhrt, so jedoch übernahm die Mutter diese Aufgabe. Daß es sich hierbei um keinen Einzelfall handelt, zeigt das Beispiel der Jeanne de Gontault, die für ihren Sohn auch in den Jahren seiner Volljährigkeit Ländereien kaufte, Verträge schloß und Prozesse fuhrte ${ }^{121}$. Anna und Henri jedenfalls unterzeichneten im April 1604 eine quittance et descharge généralle, in deren Rahmen der Prinzessin garantiert wurde, für ihre Verwaltung nicht haften zu mulssen, während sie dem Sohn versprach, ihrerseits von jeglicher Forderung abzusehen ${ }^{122}$. Neben der Aufsicht über Henris Geschäfte im Herzogtum Genevois, dem Marquisat von Saint-Sorlin und den anderen Ländereien in Savoyen mußte sich die Prinzessin um ihre eigenen, in Frankreich gelegenen Besitztümer kümmern. Hierzu zählte der Wiederaufbau von Brücken, Mauern und Gebäuden, die während der Kriege zerstört worden waren, die Verteilung von Ämtern und Würden, der Abschluß und die Erneuerung von Pachtverträgen und Lehnseiden sowie die Aufsicht über Recht und Ordnung. Die Boten, welche ununterbrochen zwischen Paris und den Orten Nemours und Montargis, Dourdan, Lion oder Fréteval unterwegs waren, zeugen von dem Aufwand, welchen diese Verwaltungstătigkeit bedeutete ${ }^{123}$.

119 Anna d'Este an Carlo Emanuele di Savoia, 1. Aug. (1596), 28. Jan. (1597), in: AST (Cast.), Int., Let. div., Prin. div. 78.

${ }^{120}$ Anna d'Este an Carlo Emanuele di Savoia, 24. Apr. u. 19. Juli (1599), Paris, in: AST (Cast.), Int., Let. div., Prin. div. 78; DIES., Memorandum für Carlo Emanuele di Savoia nebst dessen Antworten, 24. Mai 1599, in: Ibid. PGN, cat. 2, m. 14, n. 5; Dies. an die Mitglieder der Rechnungskammer von Genevois, 7. Sept. 1603, Paris, in: Ibid. n. 3.

${ }^{121}$ KaLAS, Widow's Place, S. $531 \mathrm{f}$.

122 Übereinkunft zwischen Anna d'Este und Henri de Savoie, die Haftung für die Verwaltung seiner Güter betreffend, 3. Apr. 1604, in: AN, m.c. VIII, 563, fol. 188-190.

${ }^{123}$ Königliche Beschlüsse, die Reparatur von Gebäuden u.a. in Falaise betreffend, 2. Sept. 1599, 26. Febr. 1605, 22. Febr. 1607, in: VALOIS (Hg.), Arrêts du Conseil, Bd. 1, S. 363, Bd. 2, S. 228, 367; Anweisung von Anna d'Este an Guyon, Steuereintreiber von Montargis, den Gefängniswărter des Ortes ordentlich zu bezahlen, 17. Juni 1600, in: BnF, Fr. 3234, fol. 8r; Ernennung eines Advokaten des Konigs fuir Gisors, 8. Sept. u. 23. Dez. 1601, in: BnF, Fr. 17308, fol. 65r; Beschluß des königlichen Rates, die Vergabe von Ämtem in Montargis, Nemours u.a. betreffend, Okt. 1603, in: BnF, Dup. 857, fol. 110r; Bestätigung des durch Anna d'Este ernannten Philippe Guéroult in seinem Amt als sergentpriseur-vendeur in Torigny durch den König, 29. März 1607, in: VALOIS (Hg.), Arrêts du 
In den ersten zwanzig Jahren ihrer Ehe mit dem Herzog von Nemours war Anna eine reiche Dame, sicher zählte sie zu den wohlhabendsten des französischen Adels. Die enormen Summen, die sie beispielsweise dem König lieh, beliefen sich im Jahre 1576 bereits auf 200000 Livres. Ganze 12000 Livres zahlte sie, um einen ihrer Klienten, einen wegen Steuerhinterziehung zum Tode verurteilten Salzhändler, aus dem Gefängnis zu befreien, und als Beitrag zur Versöhnung der Eidgenossenschaft mit dem Herzog von Savoyen lud die Prinzessin die Schweizer Abgeordneten zu einem Essen, welches sie nicht weniger als tausend Crowns gekostet haben soll ${ }^{124}$. Die unendlichen Prozesse sowie die Bemühungen um die Karrieren und Heiraten der Kinder verschlangen jedoch ein Vermögen, und spätestens seit Mitte der 1580er Jahre scheint sich Anna in finanziellen Schwierigkeiten befunden zu haben, nicht anders als etwa ihr Neffe Charles, Herzog von Aumale, der sein Geld in die Finanzierung der Ligue investiert hatte und die Aufsicht über seine hoffnungslose finanzielle Situation fortan seiner Gattin Marie überlie $\beta^{125}$. Im Mai 1586 borgte Anna sich von ihrem Bruder Alfonso eine große Summe, die sie Jahre später immer noch nicht zurückgezahlt hatte, und den Herzog von Savoyen bat sie gar um 20000 Écus. Zwar hatte ihr Sohn eines seiner Häuser in Piemont an Carlo Emanuele verkauft, doch die vereinbarten Zahlungen ließen auf sich warten, und so klagte die Prinzessin, die Ausgaben, die sie für den Unterhalt ihrer Kinder getătigt habe und jeden Tag tätigen müsse seien so hoch, que nous an sommes ynfinymant an arrière ${ }^{126}$.

Die unter recette de l'aryant que y'ai recceu in den Rechnungsblichern verzeichneten Summen lassen erkennen, daß die Herzogin von Nemours ihr

Conseil, Bd. 2, S. 379; Verschiedene Pachtverträge, 1595, 1605, in: LELOUP, Anne d'Este, S. 60f. u. AN, m.c. VIII, 567; Anna d'Este an Monsieur de Thignonville, einen Pachtvertrag betreffend, 5. Apr. o.J. (1594-1595), Paris, in: BnF, Fr. 20464, S. 77; Antoinette de Pons an Anna d'Este bezilglich der Ablegung eines Lehnseides, o.D. (1590ff.), in: BnF, Fr. 3397, fol. 77r. Zu den Boten vgl. die zahlreichen Einträge in: Rechnungsbücher 1591, 1593-II.

${ }^{124}$ Henry Cobham an Francis Walsingham, 7. Dez. 1582, Paris, in: Calendar Elizabeth, 1909, S. 489f. Zu den Einnahmen der Herzöge von Nemours: Rechnungsbuch 1567 für das Herzogtum Nemours, in: AST (Piave), ACP, Art. 806, par. 2, reg. 21. Zu den Schulden des Königs: Antonio Maria Salviati an Tolomeo Galli, 11. Aug. 1576, Paris, in: Correspondance Salviati, Bd. 2, S. 504. Darïber hinaus fungierte Anna als Vermittlerin zwischen dem König und italienischen Bankiers: Robert J. KNECHT, The Rise and Fall of Renaissance France, 1483-1610, London 1996, S. 476. Zum befreiten Salzhändler: Claude HATON, Mémoires (1553-1582), Bd. 2, hg. v. Félix BOURQUELOT, Paris 1857, S. 1071 (1581).

${ }^{125}$ CARROLL, Noble Power, S. 245. Vgl. den Hinweis auf die Verschuldung anderer französischer Adliger Mitte der 1580er Jahre, in: Mark GREENGRASS, France in the Age of Henri IV. The Struggle for Stability, London, New York ${ }^{2} 1995$, S. 223 f.

${ }^{126}$ Anna d'Este an Carlo Emanuele di Savoia, 23. Sept. 1589, Paris, in: AST (Cast.), Int., Let. div., Prin. div. 78. Die Bitte um 20000 Écus in einem Brief vom Juli 1587, in: lbid. Zu den Schulden beim Bruder: Dies. an Alfonso d'Este, Mai 1586, 12. Sept. 1588, Paris, in: ASM, Canc. duc., Cart. prin. est. 1458/22. Es handelte sich um die Summe von 11600 Écus, also rund 35000 Livres. 
tăgliches Leben zu einem großen Teil mit außerordentlichen Einnahmen aus Anleihen und Verpfändungen bestritt. Allein im Jahre 1591 lieh sie sich weit über viertausend Écus, erhielt weitere zweitausend von ihrem Sohn geschenkt und veräußerte einige Stücke ihres Tafelsilbers im Wert von über siebenhundert Écus, ganz zu schweigen von den in den folgenden Jahren erfolgten Verpfändungen ${ }^{127}$. Auch begann die Prinzessin, welche sich bisher als glühende Gegnerin Spaniens ausgewiesen hatte, parlandone ordinariamente con troppo disprezzo e con molta passione, in jener Zeit dem Botschafter Philipps II. Besuche abzustatten. Den Agenten des Herzogs von Ferrara ließ der spanische Gesandte den Grund für dieses unerwartete Interesse wissen: Anna hasse zwar die Spanier, nicht aber das spanische Gold, und als Beweis habe er Quittungen in der Hand, die mit Anna di Este unterzeichnet seien ${ }^{128}$. Der Kampf um die Thronfolge in Frankreich und die immerwährenden Kriege trugen kaum zu einer Verbesserung der finanziellen Situation bei. Anfang Mai 1595 klagte die Herzogin über die désolation universelle de touttes mes terres et de la plus part de mes maisons, où mes milleurs meubles ont esté perdus, aufgrund derer sie sich in einer extremen Notlage befände ${ }^{129}$. Wieder war Anna gezwungen, sich Geld zu leihen, fur welches sie einen Diamantring verpfändete und das zurückzuzahlen, wie der Bruder erfuhr, sie nicht in der Lage war, ayant par desa recherché tous mes moyens, voyre yusques au bout, y'ai trouvé qu'il m'est du tout ynpossible d'y satisfayre. Und wieder war Alfonso es, der die Schulden der Schwester beglich ${ }^{\mathrm{130}}$.

An der allgemeinen Lage änderte dies indes nichts, vielmehr ist aus den Briefen von Bénigne Pastey, Annas Steuereintreiber und Schatzmeister in Annecy, von der desolaten Situation im Herzogtum zu erfahren. Er sei eben im Genevois angekommen, schreibt Pastey, und habe eine vollkommene Unordnung und unglaubliche Armut der Bevölkerung vorgefunden, die Prinzessin werde dieses Jahr wohl keine Einnahmen zu erwarten haben und müsse unbedingt handeln, wolle sie die Pfändung ihrer Möbel verhindern:

${ }^{127}$ Verpfändung von Schmuckstücken und Tafelsilber, in: Rechnungsbücher 1591, 1593-II u. LELOUP, Anne d'Este, S. 65f. (16. Sept. 1595). Daß die Verpfändung von Wertgegenständen eine găngige Praxis adliger Finanzpolitik war, zeigen die Beispiele in: NEUSCHEL, Nobel Households, S. 612, Anm. 33.

${ }^{128}$ Ercole Rondinelli an Alfonso d'Este, 11. Sept. 1590, Paris, in: ASM, Canc. duc., Amb. Francia 95.

${ }^{129}$ Anna d'Este an Lucrezia d'Este, 1. Mai (1595), Paris, in: ASM, Canc. duc., Cart. prin. est. 1459/23. Zu den Verlusten durch kriegsbedingte Zerstörungen im Herrschaftsgebiet der Albret: EURICH, Power, S. 16-19.

${ }^{130}$ Anna d'Este an Alfonso d'Este, 1. Mai u. 23. Sept. 1595, in: ASM, Canc. duc., Cart. prin. est. 1458/22; Zwei Verträge, diesen Vorgang betreffend, 1. Sept. 1595, in: LELOUP, Anne d'Este, S. 63; Empfangsbestătigung von Antonio Bonvisi, 26. Mai 1599, in: ASM, Casa 358. Diesmal handelte es sich um die Summe von 4000 Écus. 
Je ne veois pas qu'il soit besoin de vous en dire davantaige. Je feray seullement en cest endroict ceste humble prière à dieu de vous conseiller en telle sorte que, prenant bon conseil, vous l'exécuties pour vous tirer d'affaires, affin qu'il ne vous advienne pis et que trop tard vous ne vous repenties de n'avoir creu ${ }^{131}$.

Und Anna handelte prompt, ließ sich von ihrem Rat verschiedene Möglichkeiten zur Lősung des Problems unterbreiten und entschied dann, pour le bien et advancement des affaires de mondit filz, einige seiner Ländereien zu veräuBern. Außerdem legte sie dem Herzog von Savoyen die Situation dar, schilderte die materielle Lage ihrer Untertanen und klagte, daß sie aufgrund derer Armut fast nichts an jährlichen Einnahmen erhalte. Carlo Emanuele erwies sich als großzügig und gestand ihr und ihrem Sohn die Summe von 60000 Francs $\mathrm{zu}^{132}$.

Auf Dauer konnte jedoch auch dies die fortschreitende Verschuldung der Herzöge von Nemours nicht aufhalten. Obwohl Anna neben dem Gewinn aus ihren Ländereien über Einkünfte aus Pensionen, Ämtervergaben und den Verkauf von in ihren Wäldern geschlagenen Holzes verfügte, hatte sie Anfang des 17. Jahrhunderts jedes Jahr Ausgaben in Höhe von 10000 Écus allein für Essen und Trinken, Hafer für die Pferde und Holz fur Küche und Kamine. Hinzu kamen über zweitausend Écus für die Löhne ihrer Bediensteten, die zu bezahlen sie nicht selten jahrelang in Verzug war ${ }^{133}$. Zur Bestreitung ihrer Ausgaben war die Prinzessin gezwungen, Verneuil und einige andere ihrer Besitztümer zu verkaufen sowie weitere Perlen, Kristall und Silber zu veräuBern, um die Gegenstände dann bei Bedarf von den neuen Eigentümerinnen zu entleihen ${ }^{134}$. Ein Vergleich mit anderen Angehörigen des französischen Adels zeigt, daß die Situation der Herzöge von Nemours keinesfalls eine Besonder-

${ }^{131}$ Bénigne Pastey an Anna d'Este, 7. Dez. 1596, Annecy, in: AST (Piave), ACP, Art. 806, par. 2, reg. 104.

${ }_{132}$ ESTE, Memorandum furr Carlo Emanuele. Das Zitat: Anna d'Este an die Mitglieder der Rechnungskammer von Genevois, 22. März 1597, Paris, in: Ibid. n. 3. Zum Anstieg der Hăufigkeit von Landveräußerungen durch den Adel in dieser Zeit: Mack P. HOLT, The French Wars of Religion, 1562-1629, Cambridge 1995, S. 204.

${ }^{133} \mathrm{Zu}$ Annas Einkünften vgl. die königlichen Beschllisse, 1595-1603, in: ValoIS (Hg.), Arrêts du Conseil, Bd. 1, S. 132, 288, 306, 318, 323, 354, Bd. 2, S. 67, 87, 134. Zu den Ausgaben: Rechnungsbuch 1599-1606. Einigen ihrer demoiselles war Anna noch 1591 die Löhne für die Jahre 1588-1590 schuldig, und ihrem späteren maître d'hôtel Camille Mahy hatte sie einige ihrer Seidenstoffe als Garantie für die ausstehende Bezahlung ausgehändigt: Rechnungsbuch 1591, fol. 2r, 8v, 47r.

${ }^{134}$ Protokoll über den Verkauf von Perlen für rund 4000 1.t. und Inventare des verkauften Kristalls und des mehrmals von Madame Hotman geliehenen Silbergeschirrs nebst Annas Versprechen, es zurückzugeben, 1602-1606, in: AST (Piave), ACP, Art. 806, par. 2, reg. 151; Verpfändung von Silbergeschirr im Wert von 2000 Écus an Madame Hotman und Liste der mit dem aus dem Verkauf von Verneuil, Villiers, Saint-Paul u.a. erhaltenen Geld beglichenen Schulden, in: Rechnungsbuch 1599-1606. Vgl. den Hinweis auf den Verkauf von Tafelsilber an Madame Hotman, aus dessen Erloss nach Annas Tod ihre Schulden bei diversen Händlern und Handwerkern beglichen wurden: Anhang A, fol. 19v. 
heit darstellte. So war etwa Heinrich von Navarra zu Beginn der 1590er Jahre einem großen Teil der Mitglieder seiner maison den Lohn schuldig. Die Schulden der Nevers entstanden zu einem großen Teil durch Ausgaben fur Luxusguter und Lebensmittel, während eine vergleichbar geringe Summe in die Löhne der Angestellten floß. Auch die Mitglieder dieser Familie verschuldeten sich immer mehr, indem sie sich wiederholt größere Beträge borgten, sie beglichen ihre Rückstände durch den Verkauf von Land und, in geringerem $\mathrm{Maße}$, von in ihren Wäldern geschlagenen Holzes. Und obwohl auch der Herzog von Nevers regelmäßig mit seinem Rat tagte, um seine Schulden in den Griff zu bekommen, entwickelten sich diese ab Beginn der 1590er Jahre stetig nach oben ${ }^{135}$.

Als sich Ferdinando de' Medici im Herbst 1604, wohl in Erwägung einer möglichen Verschwägerung, über die Lebensverhältnisse der Herzöge von Nemours erkundigte, wurde ihm ein sicherlich in vielen Punkten ubertriebenes, aber doch aufschlußreiches Bild von deren Situation gezeichnet:

non è casa a Parigi di principe più disordinata, più scompigliata, più indebitata, e peggio governata di questa. La duchessa è rimbambita, $e$ in quella casa è come essere in un porcile, tanta lordura vi è; sonvi quattro o cinque servitori vecchi, che consumano tutto; i debiti sono molti ${ }^{136}$.

Kaum drei Jahre spăter, nach dem Tod der Prinzessin, sollte der Wert ihrer beweglichen Hinterlassenschaft nur wenig mehr als viertausend Livres betragen, eine verschwindend geringe Summe für eine Dame wie Anna. Tatsächlich stellten sich die mit der Inventarisierung beauftragten Notare die Frage, was mit Schmuck, Geschirr und wertvoller Kleidung geschehen sei, da sie meinten, es musse mehr vorhanden sein als das, was im Haus zu finden war. Zwar hatte die Dienerschaft einige wenige Stücke an sich genommen, vielleicht als Entschädigung für den ausstehenden Lohn, und zur Bezahlung verschiedener Pariser Händler war für rund siebenhundert Livres Tafelsilber verkauft worden, doch uber den Verbleib der restlichen Wertgegenstände konnte keiner der Anwesenden Auskunft erteilen ${ }^{137}$.

${ }^{135}$ Zu Heinrich IV.: EURICH, Power, S. 99. Zu den Nevers: CrOUZET, Crise de l'aristocratie.

136 Baccio Giovannini an Ferdinando de' Medici, 17. Okt. 1604, in: DESJARDINS (Hg.), Négotiations diplomatiques, Bd. 5, S. 549.

${ }^{137}$ Anhang A, fol. 4r, 19v, 22v, 23r. Vgl. DUBOST, France italienne, S. $289 f$. 
